TRANSACTIONS OF THE

AMERICAN MATHEMATICAL SOCIETY

Volume 349, Number 5, May 1997, Pages 1697-1724

S 0002-9947(97)01868-0

\title{
STRUCTURAL PROPERTIES OF UNIVERSAL MINIMAL DYNAMICAL SYSTEMS FOR DISCRETE SEMIGROUPS
}

\author{
BOHUSLAV BALCAR AND FRANTISEK FRANEK
}

\begin{abstract}
We show that for a discrete semigroup $S$ there exists a uniquely determined complete Boolean algebra $B(S)$ - the algebra of clopen subsets of $M(S) . M(S)$ is the phase space of the universal minimal dynamical system for $S$ and it is an extremally disconnected compact Hausdorff space. We deal with this connection of semigroups and complete Boolean algebras focusing on structural properties of these algebras. We show that $B(S)$ is either atomic or atomless; that $B(S)$ is weakly homogenous provided $S$ has a minimal left ideal; and that for countable semigroups $B(S)$ is semi-Cohen. We also present a class of what we call group-like semigroups that includes commutative semigroups, inverse semigroups, and right groups. The group reflection $G(S)$ of a grouplike semigroup $S$ can be constructed via universal minimal dynamical system for $S$ and, moreover, $B(S)$ and $B(G(S))$ are the same.
\end{abstract}

\section{INTRODUCTION}

In topological dynamics it is usual to consider an action of a group on a topological space $[\mathrm{E}],[\mathrm{G}],[\mathrm{A}],[\mathrm{deV}]$. We shall consider an action of a discrete semigroup on a compact Hausdorff space and focus our attention to universal minimal dynamical systems. For this we shall consider the semigroup $(C(X, X), \circ)$, where $C(X, X)$ is the set of all continuous functions from a topological space $X$ into itself, and the operation $\circ$ is defined by $(f \circ g)(x)=f(g(x))$ for $f, g \in C(X, X)$ and $x \in X$. From now on, unless stated otherwise, by a semigroup or a group we mean a discrete semigroup or group.

Definition 1.1. Let $S=(S, \cdot)$ be a semigroup. A dynamical system $(X, S, \pi)$ consists of

(i) a non-empty compact Hausdorff space $X$, called phase space;

(ii) an action $\pi$ of the semigroup $S$ on the space $X$; that is, a homomorphism $\pi:(S, \cdot) \rightarrow(C(X, X), \circ)$ such that if $S$ has the identity $e$, then $\pi(e)$ is the identity function on $X$.

For a given dynamical system $(X, S, \pi)$ we will omit $\pi$ and refer to the system as $(X, S)$, if no ambiguities or confusion arise. Similarly we may write $s(x)$ for

Received by the editors July 5, 1994.

1991 Mathematics Subject Classification. Primary 54H20, 03G05, Secondary 06E05.

Key words and phrases. Semigroup, group, dynamical system, minimal dynamical system, universal minimal dynamical system, ultrafilter, ultrafilter dynamical system, Boolean algebra, Cohen alegbra, extremally disconnected compact space.

The research of the first author was supported by AV ČR research grant 119401, while the research of the second author was supported by NSERC research grant OGP0025112.

(C)1997 American Mathematical Society 
$\pi(s)(x)$. Note that we do not demand that the action of $S$ be effective, i.e. that $\pi$ be an embedding, and so it is possible that $\pi(s)=\pi(t)$ while $s \neq t, s, t \in S$.

For a subset $A$ of $X$ and $s \in S, s[A]$ denotes the set $\{s(a): a \in A\}$. Unless stated otherwise, $s^{-1}$ does not refer to the inverse element of $s$ but rather to the inverse function of $s$, i.e. $s^{-1}[A]=\{x \in X: s(x) \in A\}$.

Let us recall some fundamental notions from topological dynamics.

Let $(X, S, \pi)$ be a dynamical system. A subset $Y$ of $X$ is an $S$-invariant set if $Y \neq \emptyset$ and if, for every $s \in S, \pi(s)[Y] \subseteq Y$. For a closed $S$-invariant subset $Y$ of $X$ we can restrict the action of $S$ on $X$ to an action on $Y$ by defining $\pi_{Y}(s)=\pi(s)\lceil Y$ for every $s \in S$. The resulting dynamical system $\left(Y, S, \pi_{Y}\right)$ is a subsystem of $(X, S, \pi)$. The orbit of $x \in X$ is the set $\operatorname{Orb}(x)=\{\pi(s)(x): s \in S\}$.

Definition 1.2. The system $(X, S)$ is called minimal if $X$ has no proper closed $S$-invariant subset.

It is easy to see that for a system $(X, S)$ the following are equivalent:

(i) $(X, S)$ is minimal;

(ii) the orbit of each $x \in X$ is dense in $X$;

(iii) for every non-empty open $U \subseteq X, \bigcup_{s \in S} s^{-1}[U]=X$.

Proposition 1.3. For every dynamical system $(X, S)$ there is a dynamical subsystem of $(X, S)$ that is minimal.

The proposition follows from Zorn's principle and the fact that $X$ is compact.

Definition 1.4. A homomorphism from a dynamical system $(X, S, \pi)$ into a dynamical system $(Y, S, \sigma)$ is a continuous mapping $\phi: X \rightarrow Y$ that commutes with the actions; i.e. $\phi(\pi(s)(x))=\sigma(s)(\phi(x))$ for all $s \in S$ and all $x \in X$. If moreover $\phi$ is a homeomorphism, then $\phi$ is called an isomorphism. $(Y, S, \sigma)$ is a factor of $(X, S, \pi)$ if there is a homomorphism from $(X, S, \pi)$ onto $(Y, S, \sigma)$.

Lemma 1.5.

(1) If $\phi:(X, S) \rightarrow(Y, S)$ is a homomorphism and $(Y, S)$ is minimal, then $\phi$ must be onto.

(2) A factor of a minimal system is also minimal.

Definition 1.6. A system $(X, S)$ is called a universal minimal dynamical system if it is minimal and every minimal dynamical system for $S$ is a factor of $(X, S)$.

For every discrete semigroup there is a unique (up to isomorphism) universal minimal dynamical system [E]. It has been known that for a group the phase space of a universal minimal system is an extremally disconnected compact Hausdorff space (Ellis - see $[\mathrm{vD}]$, for countable groups see $[\mathrm{Ga}]$ ). We prove that the same holds true for semigroups (Theorem 3.3).

This was the motivation for studying extremally disconnected dynamical systems in [BD], in particular two natural dynamical systems: $(X, \operatorname{Hom}(X))$ and $(X, \operatorname{Emb}(X))$, where $X$ is an extremally disconnected compact Hausdorff space, $\operatorname{Hom}(X)$ is the group of all homeomorphisms of $X$, and $\operatorname{Emb}(X)$ is the semigroup of all continuous embeddings of $X$ into itself. Among other results the conditions when these systems are minimal were presented. In Example 3.4 (ii) we observe that $(X, E m b(X))$ is not universal minimal for any infinite $X$. It is likely that the same is true for the system $(X, \operatorname{Hom}(X))$, but the problem is still unresolved. 
Since the phase space, denoted $M(S)$, of a universal minimal dynamical system for a semigroup $S$ is an extremally disconnected compact Hausdorff space, the Boolean algebra of clopen subsets of $M(S)$ is complete. We shall denote it by $B(S)$. Our aim is to investigate the structural properties of these algebras for various classes of semigroups. Let us remark here that the universal minimal dynamical system for a semigroup $S$ is given not only by its phase space, but also by the way $S$ acts on it. Therefore, it is possible that for different semigroups the respective phase spaces of their universal minimal dynamical systems are the same and the systems only differ in the way the semigroups act on it.

It is known that a group acts effectively on the phase space of its universal minimal dynamical system [E]. In general, though, a semigroup $S$ may not act effectively on $M(S)$. The action of $S$ on $M(S)$ defines a congruence $\rho(S)$ on $S$ : $(s, t) \in \rho(S)$ iff $s(x)=t(x)$ for all $x \in M(S)$. That $\rho(S)$ is well defined will be shown later (see Remark 3.2).

In summary, through the universal minimal dynamical system we have associated with each semigroup $S$

- a uniquely determined extremally disconnected compact Hausdorff space $M(S)$;

- a uniquely determined complete Boolean algebra $B(S)$;

- a uniquely determined congruence $\rho(S)$ on $S$ which expresses how effectively $S$ acts on $M(S)$;

- a uniquely determined group Aut $(S)$ of automorphisms of the universal minimal dynamical system for $S$.

Since we shall make frequent references to free, Cohen, and semi-Cohen Boolean algebras, let us present related definitions and notions at this point.

For an infinite cardinal $\kappa, \mathbb{C}(\kappa)$ denotes one of the basic types of complete Boolean algebras, namely the completion of the free algebra with $\kappa$ generators. A Boolean algebra $\mathcal{B}$ is a regular subalgebra of a Boolean algebra $\mathcal{A}$ if every subset of $\mathcal{B}$ that has a join in $\mathcal{B}$ has the same join in $\mathcal{A}$, or, equivalently, if every maximal antichain in $\mathcal{B}$ (i.e. a maximal pairwise disjoint family of non-zero elements of $\mathcal{B}$ ) is a maximal antichain in $\mathcal{A}$. For a set $X,[X]^{\omega}$ denotes the set of all countable subsets of $X$. $Y \subseteq[X]^{\omega}$ is closed unbounded (or club for short) if (a) for any $x \in[X]^{\omega}$ there is $y \in Y$ such that $x \subseteq y$ and (b) $Y$ is closed under unions of chains of countable length. The notion of semi-Cohen algebra is due to S. Fuchino and T. Jech. In [Ko1] Cohen algebra is defined in a similar way.

Definition 1.7. An infinite complete Boolean algebra $\mathcal{B}$ of uniform density is called semi-Cohen if the set $\mathcal{S}=\left\{A \in[B]^{\omega}: A\right.$ is a regular subalgebra of $\left.\mathcal{B}\right\}$ contains a club. It is called a Cohen algebra if the set $\mathcal{S}$ contains a club $\mathcal{C}$ with the property that if $\mathcal{A}_{1}, \mathcal{A}_{2} \in \mathcal{C}$, then the subalgebra of $\mathcal{B}$ generated by $\mathcal{A}_{1} \cup \mathcal{A}_{2}$ and denoted by $\left\langle\mathcal{A}_{1} \cup \mathcal{A}_{2}\right\rangle$ is also in $\mathcal{C}$.

See Proposition 6.1 for the mutual relationship of free, semi-Cohen, and Cohen algebras. For more on semi-Cohen and Cohen algebras see [BJZ].

Among other results concerning the structural properties of the Boolean algebra $B(S)$ associated with a discrete semigroup $S$ we prove that:

- $B(S)$ is isomorphic to $B(I)$ for any left ideal $I$ of $S$ (Theorem 3.5);

- $B(S)$ is weakly $\pi$-homogeneous (Proposition 5.1);

- $B(S)$ is either atomic or atomless (Proposition 5.4); 
- $B(S)$ is weakly homogeneous if $S$ has a minimal left ideal (Theorem 5.7);

- an infinite $B(S)$ is homogeneous if $S$ is left cancellative (Theorem 5.7);

- if a countable $S$ is left cancellative, or has a minimal left ideal, or is commutative, then $B(S)$ is either atomic or a Cohen algebra (Theorem 6.2);

- if $S$ is countable, then the algebra $B(S)$ is either $\mathcal{P}(X)$ for some at most countable set $X$ or a semi-Cohen algebra completely embeddable in a Cohen algebra (Theorem 6.6);

- if $B(S)$ is atomic, then $A u t(S)$ is finite (Proposition 8.3).

It is well known [HS] that semigroups have group reflections, i.e. that for any semigroup $S$ there are a group $G(S)$ and a homomorphism $\varphi: S \rightarrow G(S)$ so that if $H$ is an arbitrary group and $\phi: S \rightarrow H$ a homomorphism, then there exists a unique homomorphism $\xi: G(S) \rightarrow H$ so that $\xi \circ \varphi=\phi$. It follows that $G(S)$ is generated by $\varphi[S]$ and is also unique (up to isomorphism). In $[\mathrm{L}]$ an explicit construction of the group reflection $G(S)$ of a commutative monoid $S$ is given and is called the Grothendieck group for $S$. In general if $G(S)$ is a reflection of $S$, then the universal minimal dynamical system for $S$ and the universal minimal dynamical system for $G(S)$ need not necessarily be the same; e.g. if $S$ is a left zero semigroup, then its reflection $G(S)$ is a trivial group. On the other hand (see Example 4.8), $B(S) \cong \mathcal{P}(S)$. We shall show, though, that for the class of what we call group-like semigroups (see section 7 ) that includes commutative semigroups, inverse semigroups, and right groups, the group $G(S)$ reflecting such an $S$ can be obtained via its universal minimal dynamical system and, moreover, that the universal minimal dynamical system for $S$ is the same as the universal minimal dynamical system for $G(S)$.

We would like to turn the reader's attention to a few basic open problems:

- It is known that if $S$ is a group, then it acts effectively on its universal minimal dynamical system $(M, S, \pi)$, i.e. that the congruence $\rho(S)=i d_{S}$, or equivalently, that $\pi(s) \neq \pi(t)$ whenever $s \neq t \in S$. This is also true for instance for left zero semigroups. Is there a relatively simple characterization of semigroups that act effectively on their universal minimal dynamical systems?

- We show (see 5.7) that for several classes of semigroups, $B(S)$ is weakly homogeneous for such an $S$. We conjecture that this is true for all semigroups.

- For a countable $S, B(S)$ is a complete subalgebra of the Cohen algebra $\mathbb{C}\left(2^{\omega}\right)$. Thus, under $\mathrm{CH}, B(S)$ is either isomorphic to a power set of a countable set, or the Cohen algebra $\mathbb{C}(\kappa)$ for some $\kappa \leqslant 2^{\omega}$ (Corollary 6.7). We conjecture that this holds true without any set-theoretical assumptions. Let us remark that the problem whether a complete subalgebra of a Cohen algebra is again a Cohen algebra had been open for years. Recently Koppelberg and Shelah $[\mathrm{KS}]$ constructed a complete subalgebra of $\mathbb{C}\left(\omega_{2}\right)$ that is not Cohen.

We are indebted to the referee of this paper for bringing to our attention an interesting paper [HiS] where a few somehow similar results stated for commutative discrete semigroups are presented. In particular, the result of Proposition 8.1 can be found there (Theorem 3 in $[\mathrm{HiS}]$ ). Though stated for commutative semigroups, the proof goes along the same lines as our proof using Frolík's theorem. The fact (stated for general semigroups here in Theorem 3.3) that $M(S)$ must be extremally disconnected for a commutative semigroup $S$ is also presented in [HiS]. 


\section{Preliminaries}

Let us recall basic notions and facts from the theory of semigroups, topological spaces, and Boolean algebras.

(a) Semigroups. For an overview of the theory of semigroups see $[\mathrm{CP}]$. Let $S=(S, \cdot)$ be a semigroup.

An element $x \in S$ is an idempotent if $x \cdot x=x . \operatorname{Id}(S)$ denotes the set of all idempotents of $S$. An element $x \in S$ is a left (right) zero if $x \cdot y=x(y \cdot x=x)$ for every $y \in S$. Elements $x, y \in S$ are said to be inverse if $x=x \cdot y \cdot x$ and $y=y \cdot x \cdot y$. $S$ is called an inverse semigroup if every $x \in S$ has a unique inverse element. $S$ is called a left (right) zero semigroup if each element of $S$ is a left (right) zero. $S$ is called left (right) cancellative if $a \cdot x=a \cdot y(x \cdot a=y \cdot a)$ implies $x=y$ for any $a, x, y \in S$. A non-empty subset $I$ of $S$ is a left (right) ideal of $S$ if $S \cdot I \subseteq I(I \cdot S \subseteq I)$. $S$ is called left (right) simple if it has no proper left (right) ideal. $S$ is called a left (right) group if it is left (right) simple and right (left) cancellative. For $s \in S$, the mapping $L_{s}: S \rightarrow S$ defined by $L_{s}(t)=s \cdot t$ is called left translation of $s$ (sometimes denoted also as $l_{s}$ ), and the mapping $R_{s}: S \rightarrow S$ defined by $R_{s}(t)=t \cdot s$ is called right translation of $s$ (sometimes denoted also as $r_{s}$ ).

The following structural theorem for semigroups can be found in [CP].

Theorem 2.1. For a semigroup $S$, the following are equivalent:

(1) $S$ is left simple and has an idempotent;

(2) $S$ is a left group, i.e. $S$ is left simple and right cancellative;

(3) $S$ is a direct product $G \times E$ of a group $G$ and a left zero semigroup $E$.

Note that (3) in fact says the following: $S$ is a disjoint union of mutually isomorphic groups $\{G(e): e \in I d(S)\}$, where $\operatorname{Id}(S)$ is the set of all idempotents of $S$. For each $e \in \operatorname{Id}(S), e$ is the identity of $G(e)$. Let $e_{1}, e_{2} \in \operatorname{Id}(S)$. Every $y \in G\left(e_{2}\right)$ has a unique element $y^{\prime} \in G\left(e_{1}\right)$ "corresponding" to $y$ in the sense that if $x \in G\left(e_{1}\right)$, then $x \cdot y=x \cdot y^{\prime}$. In particular $e_{1} \cdot e_{2}=e_{1}$. Thus, $G\left(e_{1}\right) \cdot G\left(e_{2}\right)=G\left(e_{1}\right)$. We shall call the groups $G(e)$ 's the partition groups of $S$.

(b) One-sided topological semigroups. A semigroup $S=(S, \cdot)$ together with a topology $\tau$ on the set $S$ is a right-topological compact semigroup if $\tau$ is a compact Hausdorff topology on $S$ and for every $t \in S$, the right translation $R_{t}$ is continuous.

A well-known and important fact (see $[\mathrm{E}],[\mathrm{G}],[\mathrm{F}],[\mathrm{A}],[\mathrm{deV}]$ ) is

Theorem 2.2 (Numakura). A right-topological compact semigroup has an idempotent.

Consider a right-topological compact semigroup $S$ and a left ideal $I$ of $S$. For any $t \in I, S \cdot t \subseteq I$ and, moreover, $S \cdot t$ is a topologically closed ideal of $S$. Therefore, by Zorn's principle, there is a minimal left ideal of $S$ and every such ideal is topologically closed.

Let $M$ be a minimal left ideal of $S$. It follows that $(M, \cdot)$ is a right-topological compact semigroup, and therefore it has an idempotent. Consequently, it has the algebraic structure as described in Theorem 2.1. Moreover, if $M_{1}$ and $M_{2}$ are two distinct minimal left ideals of $S$, then they are necessarily disjoint and for every idempotent $e \in M_{1}, R_{e}\left\lceil M_{2}: M_{2} \rightarrow M_{1}\right.$ is a homeomorphism. Furthermore, for any $e \in \operatorname{Id}\left(M_{1}\right)$ there exists a unique $j \in M_{2}$ so that $e \cdot j=j$ and $j \cdot e=e$. 
(c) Topological spaces. For an overview of general topology see [En], for the topology concerning Boolean spaces see [BS]. A topological space for us is a Hausdorff space. Recall that a space is extremally disconnected if the closure of every open set is again open, and therefore clopen. It follows that every extremally disconnected compact space is zero-dimensional as it has a base that consists of clopen sets. In an extremally disconnected compact space $X$ regular open and clopen subsets of $X$ are the same, so the field of clopen subsets of $X, C O(X)$, is a complete Boolean algebra as it is equal to the complete Boolean algebra of all regular open subsets of $X, R O(X)$.

A mapping $f$ from a space $X$ into a space $Y$ is open if $f[U]$ is open for any open $U \subseteq X$, and is semiopen if $f[U]$ has a non-empty interior for any non-empty open $U \subseteq X$. A continuous mapping $f: X \rightarrow X$ is a retraction on $X$ if $f \circ f=f$. A subspace $Y$ of $X$ is a retract of $X$ if it is the range of some retraction on $X$.

The following proposition is well known.

Proposition 2.3. A retract of an extremally disconnected Hausdorff space $X$ is a closed extremally disconnected Hausdorff subspace of $X$.

(d) Boolean algebras and topological duality. For an overview of the theory of Boolean algebras see $[\mathrm{H}]$. A Boolean algebra $\mathcal{B}$ is homogeneous if, given $u, v \in$ $\mathcal{B}-\{\mathbb{O}, \mathbb{I}\}$, there is an automorphism of $\mathcal{B}$ which takes $u$ to $v$. $\mathcal{B}$ is called weakly homogeneous if, given $u, v \in \mathcal{B}-\{\mathbb{O}\}$, there is an automorphism $h$ of $\mathcal{B}$ such that $h(u) \wedge v \neq \mathbb{O}$. Let us remark that an infinite Boolean algebra $\mathcal{B}$ is homogeneous iff $\mathcal{B} \cong \mathcal{B}\lceil u$ for any $u \in \mathcal{B}-\{\mathbb{O}\}$, where $\mathcal{B}\lceil u=\{u \wedge v: v \in \mathcal{B}\}$.

The next one is a fundamental and deep result due to Koppelberg [Ko] and Solovay (unpublished), independently. The proof following Solovay's approach can be found in $[\mathrm{H}]$, pp. 687-693.

Theorem 2.4 (Koppelberg-Solovay). A complete Boolean algebra $\mathcal{B}$ is weakly homogeneous iff there are a homogeneous complete Boolean algebra $\mathcal{A}$ and an index set $I$ such that $\mathcal{B} \cong \prod_{i \in I} \mathcal{A}$.

We shall call a zero-dimensional compact topological space a Boolean space, because these spaces are exactly Stone spaces of Boolean algebras. It is well known that a compact space is extremally disconnected iff it is the Stone space of a complete Boolean algebra.

Proposition 2.5. Let $X, Y$ be Boolean spaces, $\mathcal{A}=C O(X), \mathcal{B}=C O(Y)$, $f: X \rightarrow Y$ a continuous mapping and $h: \mathcal{B} \rightarrow \mathcal{A}$ the homomorphism dual to $f$. Then

(1) $f$ is semiopen iff $h$ is regular, i.e. whenever $b=\bigvee_{i \in I} b_{i}$ in $\mathcal{B}$, then $h(b)=$ $\bigvee_{i \in I} h\left(b_{i}\right)$ in $\mathcal{A}$, and hence $h[\mathcal{B}]$ is a regular subalgebra of $\mathcal{A}$.

(2) $f$ is open iff $h$ has the following property: for every $a \in \mathcal{A}$ there is the least $m(a) \in \mathcal{B}$ such that $h(m(a)) \geq a$.

In general $m(a \vee b)=m(a) \vee m(b)$ and $m(a \wedge b) \leqslant m(a) \wedge m(b)$ for any $a, b \in \mathcal{A}$.

If in addition $f$ is one-to-one (or, equivalently, if $h$ is onto), then $m(a \wedge b)=$ $m(a) \wedge m(b)$ for any $a, b \in \mathcal{A}$.

(3) If $f$ is semiopen and $Y$ is extremally disconnected, then $f$ is open. 
(4) If $f$ is open and onto, then $Y$ is extremally disconnected whenever $X$ is extremally disconnected.

Corollary 2.6. Let $X$ be an extremally disconnected Boolean space and let $\mathcal{A}=$ $C O(X)$. Then a continuous $f: X \rightarrow X$ is open iff the dual homomorphism $h$ : $\mathcal{A} \rightarrow \mathcal{A}$ is a complete homomorphism.

If $\mathcal{B}$ is a Boolean algebra and $\left\{h_{s}: s \in S\right\}$ a system of autohomomorphisms of $\mathcal{B}$ such that $h_{s} \circ h_{t}=h_{t \cdot s}$, then $\left(\mathcal{B},\left\{h_{s}: s \in S\right\}\right)$ form a Boolean dynamical system. The system is minimal iff for any $u \in \mathcal{B}-\left\{\mathbb{O}_{\mathcal{B}}\right\}$ there are a positive integer $n$ and $s_{0}$, $\ldots, s_{n} \in S$ so that $\mathbb{I}_{\mathcal{B}}=\bigvee_{i \leqslant n} h_{s_{i}}(u)$. It is a straightforward application of topological duality of Boolean algebras to see that if $(X, S)$ a minimal dynamical system with $X$ a Boolean space, then $\left(C O(X),\left\{h_{s}: s \in S\right\}\right)$, where $h_{s}(A)=s^{-1}[A]$ for every $A \in C O(X)$, is a Boolean minimal dynamical system.

On the other hand if $\left(\mathcal{B},\left\{h_{s}: s \in S\right\}\right)$ is a Boolean minimal dynamical system, then $\left(U l(\mathcal{B}),\left\{f_{s}: s \in S\right\}\right)$, where $U l(\mathcal{B})$ is the Stone space of $\mathcal{B}$, i.e. the space of all ultrafilters on $\mathcal{B}$ with the usual topology, and where $f_{s}(p)=\left\{b \in \mathcal{B}: h_{s}(b) \in p\right\}$ for every $p \in U l(\mathcal{B})$, is a minimal dynamical system. It follows that if $\left(\mathcal{B},\left\{h_{s}: s \in S\right\}\right)$ is a Boolean version of a universal minimal dynamical system and $\left(\mathcal{A},\left\{g_{s}: s \in S\right\}\right)$ is a Boolean minimal dynamical system, then there exists an embedding $\phi: \mathcal{A} \rightarrow \mathcal{B}$ that commutes with the homomorphisms $h_{s}$ 's and $g_{s}$ 's.

\section{The complete Boolean algebras associated with Semigroups}

We shall associate with every semigroup $S$ a complete Boolean algebra $B(S)$, using the phase space $M(S)$ of the universal minimal dynamical system for $S$. By the definition $B(S)$ is a Boolean algebra isomorphic to the algebra of clopen subsets of $M(S)$. First we present fundamental theorems concerning universal minimal dynamical systems. Their proofs will be presented during the description of the ultrafilter dynamical system later in this section.

Theorem 3.1 (Ellis). For every semigroup $S$, there is a unique (up to isomorphism) universal minimal dynamical system $\left(M(S), S, \pi_{S}\right)$.

Remark 3.2. Let $(M, S, \pi)$ be a universal minimal dynamical system for $S$. In section 1 we introduced the congruence $\rho(S)$ on $S$ defined by $(s, t) \in \rho(S)$ if $\pi(s)=$ $\pi(t), s, t \in S$. The definition is sound, for if $\left(M_{1}, S, \pi_{1}\right)$ is a universal minimal dynamical system for $S$, by Theorem 3.1 there is an isomorphism $\phi:(M, S, \pi) \rightarrow$ $\left(M_{1}, S, \pi_{1}\right)$. Then $\phi(\pi(s)(x))=\pi_{1}(s)(\phi(x))$ for any $x \in M$. Hence if $\pi(s)=\pi(t)$, then $\phi(\pi(s)(x))=\phi(\pi(t)(x))$, and so $\pi_{1}(s)(\phi(x))=\pi_{1}(t)(\phi(x))$. It follows that $\pi_{1}(s)=\pi_{1}(t)$.

Theorem 3.3. For any semigroup $S, M(S)$ is an extremally disconnected compact space, and for any $s \in S, \pi_{S}(s): M(S) \rightarrow M(S)$ is an open mapping.

Let us remark, that when $S$ is a group, then $S$ acts in any dynamical system via homeomorphisms, and hence open mappings. For semigroups this is no longer true in general. We now present examples of semigroups and minimal dynamical systems in which the semigroups act via mappings that are not open.

Example 3.4. (i) Consider $I$, the closed unit interval of real numbers, and let $\left\{a_{n}: n \in \omega\right\}$ be a dense subset of $I$. For each $n \in \omega$, let $f_{n}: I \rightarrow\left\{a_{n}\right\}$. This forms a minimal dynamical system for any countable left zero semigroup. 
(ii) Consider $(X, E m b(X))$ for an infinite extremally disconnected compact space $X$, where $\operatorname{Emb}(X)$ is the semigroup of all continuous embeddings of $X$ into itself. On the one hand, it is shown in [BD] that $(X, E m b(X))$ is minimal iff $X$ is homogeneous in weight. On the other hand, it is proven in $[\mathrm{BF}]$ (can also be found in $[\mathrm{H}]$ ), that every infinite extremally disconnected compact space $X$ can be embedded onto a nowhere-dense subset of $X$, hence in light of Theorem 3.3, $(X, \operatorname{Emb}(X))$ can never be universal.

Theorem 3.5. For any semigroup $S$ and left ideal $I$ of $S, M(S)=M(I)$. More precisely

(1) If $(M, S, \pi)$ is a universal minimal dynamical system for $S$, then $(M, I, \pi\lceil I)$ is a universal minimal dynamical system for $I$.

(2) If $(M, I, \pi)$ is a universal minimal dynamical system for $I$, then $\pi: I \rightarrow$ $C(M, M)$ can be extended to $\hat{\pi}: S \rightarrow C(M, M)$ so that $(M, S, \hat{\pi})$ is a universal minimal dynamical system for $S$.

In the proofs of Theorems 3.1 and 3.3 we follow Ellis' way and use extensively what we call ultrafilter dynamical system $[\mathrm{E}],[\mathrm{G}],[\mathrm{Hi}]$.

(a) Ultrafilter dynamical system. Let $S=(S, \cdot)$ be a semigroup. Let us recall that $\beta S$, the Stone-Cech compactification of the discrete set $S$, is the Stone space of the complete Boolean algebra of all subsets of $S, \mathcal{P}(S)$. As usual we consider the elements of $\beta S$ to be ultrafilters over $S$ and the topology is given by the base consisting of clopen sets of the form $A^{*}=\{p \in \beta S: A \in p\}, A \subseteq S$. We identify the principal ultrafilter determined by an element $s \in S$ with this element, therefore the discrete space $S$ is a dense subset of the space $\beta S$.

$\beta S$ is a compact Hausdorff space and $S=(S, \cdot)$ acts on $\beta S$ in a natural way: for a given $s \in S$, the left translation $l_{s}: S \rightarrow S$ has a unique continuous extension $L_{s}: \beta S \rightarrow \beta S$. It follows that $L_{s \cdot t}=L_{s} \circ L_{t}$, for any $s, t \in S$, therefore $\left(\beta S, S, \pi_{u}\right)$, where $\pi_{u}(s)=L_{s}$ for any $s \in S$, is a dynamical system for $S$. We call it the ultrafilter dynamical system for $S$.

Since the action of $S$ on $\beta S$ is now fixed, according to our convention we use $(\beta S, S)$ to denote the system, and $s(p)$ instead of $\pi_{u}(s)(p)$, for any $s \in S$ and $p \in \beta S$. Note that in combinatorial terms, $s(p)=\left\{A \subseteq S: l_{s}^{-1}[A] \in p\right\}$, for $s \in S$ and $p \in \beta S$. Since $s\left[A^{*}\right]=(s \cdot A)^{*}$ for any $A \subseteq S$, we have

Fact 3.6. $(\beta S, S)$ is a dynamical system with an extremally disconnected phase space and in which $S$ acts via open mappings.

(b) The universal minimal dynamical system. We know from Proposition 1.3 that there exists a minimal $S$-invariant closed subset $M$ of $\beta S$, and thus, $(M, S)$ is a minimal dynamical system that is a subsystem of $(\beta S, S)$.

Proposition 3.7. If $(M, S)$ is a minimal dynamical subsystem of $(\beta S, S)$, then $(M, S)$ is a universal minimal dynamical system for $S$.

Proof. Consider an arbitrary minimal dynamical system $(X, S)$. It suffices to find a homomorphism $\phi$ from $(\beta S, S)$ into $(X, S)$, because $\phi\lceil M$ will be a homomorphism from $(M, S)$ onto $(X, S)$ by Lemma 1.5 .

Fix an $x \in X$ and define $\varphi: S \rightarrow \operatorname{Orb}(x)$ by $\varphi(s)=s(x)$. There exists a unique continuous extension $\phi: \beta S \rightarrow X=\operatorname{cl}(\operatorname{Orb}(x))$ of $\varphi$. It is the desired homomorphism from $(\beta S, S)$ onto $(X, S)$. 
(c) Ultrafilter systems and right-topological semigroups. As mentioned above, $S$ is a dense subset of $\beta S$. The operation - on $S$ can be extended on the whole $\beta S$. Namely, for $p, q \in \beta S$ we define $p \cdot q=p$-lim $\langle s(q): s \in S\rangle$; it means that for any neighborhood $V$ of the point $p \cdot q$ in $\beta S$, the set $\{s \in S: s(q) \in V\}$ is in the ultrafilter $p$. Given the fact that the topology of $\beta S$ is given by a base that consists of clopen sets $A^{*}, A \subseteq S$, it follows that in combinatorial terms $p \cdot q=\left\{A \subseteq S:\left\{s \in S: s(q) \in A^{*}\right\} \in p\right\}=\left\{A \subseteq S:\left\{s \in S: l_{s}^{-1}[A] \in q\right\} \in p\right\}$.

It is easy to verify that $\cdot$ is an associative binary operation on $\beta S$ extending the operation - on $S$. The extension - has the property that for any $r \in \beta S, R_{r}: \beta S \rightarrow$ $\beta S$ is continuous, i.e. that $(\beta S, \cdot)$ is a right-topological compact semigroup. As far as the left translations are concerned, for any $s \in S, L_{s}$ is also a continuous function on $\beta S$, but generally for $p \in \beta S, L_{p}$ need not be continuous. According to paragraph (b), section 2 , there is a minimal left ideal of $(\beta S, \cdot)$ and as such it is both topologically closed and an $S$-invariant subset of $\beta S$. It follows that minimal left ideals of $(\beta S, \cdot)$ are exactly minimal closed $S$-invariant subsets of $\beta S$. Thus, minimal left ideals of $(\beta S, \cdot)$ are exactly phase spaces of minimal ultrafilter systems for $S$. Consequently, for every minimal ultrafilter system $(M, S),(M, \cdot)$ is a righttopological compact semigroup with an idempotent, and so $M$ is a disjoint union of partitions groups as described in paragraph (b), section 2 .

Lemma 3.8. Let $(M, S)$ be a minimal subsystem of the ultrafilter system for $S$. Then

(1) for every $e \in I d(M), R_{e}$ is a retraction on $\beta S$ and its range is $M$;

(2) for every $p \in M, R_{p} \uparrow M$ is an automorphism of $(M, S)$;

(3) every autohomomorphism of $(M, S)$ is of the form $R_{p}\lceil M$ for some $p \in M$, and hence an automorphism.

Proof. (1) Fix $e \in I d(M)$. Let $p \in \beta S$. Then $\left(R_{e} \circ R_{e}\right)(p)=R_{e}\left(R_{e}(p)\right)=$ $R_{e}(p \cdot e)=(p \cdot e) \cdot e=p \cdot(e \cdot e)=p \cdot e=R_{e}(p)$. Thus, $R_{e}$ is a retraction on $\beta S$. Since $M$ is a minimal left ideal of $(\beta S, \cdot), M=\beta S \cdot q$ for any $q \in M$, in particular $M=\beta S \cdot e=R_{e}[\beta S]$. Therefore $M$ is a retract and $R_{e}\left\lceil M=i d_{M}\right.$.

(2) Fix $p \in M . \quad R_{p}$ is a homomorphism from $(\beta S, S)$ into $(M, S)$ because $R_{p}(s(q))=(s \cdot q) \cdot p=s \cdot(q \cdot p)=s\left(R_{p}(q)\right)$. It follows that $R_{p}\lceil M$ is a homomorphism from $M$ onto $M$ by Lemma 1.5. That $R_{p}$ is one-to-one follows from the right cancellativness of $M$ (by Theorem $2.1(2)$ ).

(3) Let $\phi: M \rightarrow M$ be a homomorphism. Fix an $e \in I d(M)$. We shall show that $\phi=R_{\phi(e)}\left\lceil M\right.$. $\left(\phi \circ R_{e}\right)$ is a homomorphism from $(\beta S, S)$ onto $(M, S)$, and so for any $s \in S\left(\phi \circ R_{e}\right)(s)=\phi(s \cdot e)=s \cdot \phi(e)=R_{\phi(e)}(s)$. Since $S$ is dense in $\beta S$, $\phi \circ R_{e}=R_{\phi(e)}$ on the whole $\beta S$. Consequently, $\left(\phi \circ R_{e}\right)\left\lceil M=\phi=R_{\phi(e)}\lceil M\right.$.

Fact 3.9. For any $e \in I d(M(S))$, the partition group e.M(S) is isomorphic to Aut $(S)$, the group of automorphisms of $(M(S), S)$. For by Lemma $3.8(3)$, if $\phi$ is an automorphism of $(M(S), S)$, then there is $p \in M(S)$ so that $\phi=R_{p} \uparrow M(S)$. If $p \notin e \cdot M(S)$, then there is $p^{\prime} \in e \cdot M$ "corresponding" to $p$ (see the note after Theorem 2.1) so that $R_{p}=R_{p^{\prime}}$.

At this point we can present proofs of Theorem 3.1, Theorem 3.3, and Theorem 3.5 .

Proof of Theorem 3.1. If $\left(M_{1}, S\right)$ and $\left(M_{2}, S\right)$ are two minimal dynamical subsystems of the ultrafilter dynamical system for $S$, then they are isomorphic. For if 
$e \in \operatorname{Id}\left(M_{1}\right)$, then $R_{e}\left\lceil M_{2}\right.$ is an isomorphism from $\left(M_{2}, S\right)$ onto $\left(M_{1}, S\right)$. Therefore, it suffices to show that any universal minimal dynamical system $(X, S, \pi)$ is isomorphic to a minimal subsystems of the ultrafilter system for $S$.

Let $(X, S, \pi)$ be a universal minimal dynamical system, and let $\left(M, S, \pi_{u}\right)$ be a minimal subsystem of the ultrafilter system. Hence there is a homomorphism $\phi$ from $(X, S, \pi)$ onto $\left(M, S, \pi_{u}\right)$ and a homomorphism $\xi:\left(M, S, \pi_{u}\right) \rightarrow(X, S, \pi)$. Thus, $\phi \circ \xi$ is an autohomomorphism of $\left(M, S, \pi_{u}\right)$ and by Lemma 3.8 it is an automorphism of $\left(M, S, \pi_{u}\right)$. It follows that both $\phi$ and $\xi$ are isomorphisms.

Proof of Theorem 3.3. Consider a universal minimal dynamical system $(M, S)$ as a subsystem of the ultrafilter dynamical system. Choose an arbitrary $e \in \operatorname{Id}(M)$ and consider $R_{e}$. By Lemma $3.8(1), R_{e}$ is a retraction on $\beta S$ with the range $M$, i.e. $M$ is a retract of an extremally disconnected compact Hausdorff space $\beta S$. By Proposition 2.3, $M$ is also an extremally disconnected compact Hausdorff space.

To prove that $s: M \rightarrow M$ is open, we have to first prove the following claim.

Claim. Let $R: X \rightarrow X$ be a retraction of an extremally disconnected Hausdorff space $X$, and let $Y$ be its range. Let $f: X \rightarrow X$ be a continuous open mapping so that $R$ and $f$ commute, i.e. $R \circ f=f \circ R$. Then $f\lceil Y$ is an open continuous mapping from $Y$ into $Y$.

Proof of Claim. Let $W$ be an open subset of $Y$. Then $V=R^{-1}[W]$ is an open subset of $X$, and $V \cap Y=W$ since $R$ is a retraction on $X$ with the range $Y$, which makes $R\left\lceil Y=i d_{Y}\right.$. Since $f[Y] \subseteq Y$ we have $f[W]=f[V \cap Y] \subseteq f[V] \cap Y$. On the other hand, $f[V] \cap Y \subseteq f[W]$, for if $y \in f[V] \cap Y$, then there is an $x \in V$ so that $f(x)=y$, and $y=R(f(x))=f(R(x))$. As $R(x) \in W$, it follows that $y \in f[W]$.

Thus, $f[W]=f[V] \cap Y$. Since $f$ is open, $f[V]$ is an open subset of $X$, making $f[W]$ an open subset of $Y$. This completes the proof of Claim.

Due to associativity of the multiplication of ultrafilters, we have that for any $s \in S$, $R_{e} \circ s=s \circ R_{e}$. By Fact 3.6, $s$ is an open mapping from $\beta S$ into $\beta S$, and thus, by Claim, $s \uparrow M$ is an open mapping from $M$ into $M$.

Example 3.10. Let $S=(S, \cdot)$ be a semigroup with a left zero $z$. Let $A=S \cdot z$. Then $S \cdot A=A$ and therefore $A$ is a left ideal of $S$. $A$ is also the set of all left zeros of $S$ and $(A, \cdot)$ a left zero semigroup. According to Theorem $3.5, B(S) \cong B(A)$. Nonetheless, for this example this fact can be observed directly:

Every $S$-invariant subset of $\beta S$ includes $A^{*}$. Thus, $\beta A$ is a minimal closed $S$ invariant subset of $\beta S$, i.e. $(\beta A, S)$ is a universal minimal dynamical system for $S$. In particular:

Let $A$ be a non-empty set, and let $S=\left({ }^{A} A, \circ\right)$ be the semigroup of all functions from $A$ into itself with the operation of composition. $(\beta A, S)$ is a universal minimal dynamical system for $S$, as the set of all constant functions on $A$ is the set of all left zeros of $S$.

Proof of Theorem 3.5. (1) If $p \in \beta I$, then $\hat{p}=\{A \subseteq S:(\exists B \in p)(B \subseteq A)\}$ is a uniquely determined ultrafilter over $S$. As usual we identify $p$ and $\hat{p}$, and in this sense $\beta I \subseteq \beta S$. Moreover, $(\beta I, \cdot)$ is a subsemigroup of $(\beta S, \cdot)$. Since $S \cdot I \subseteq I$, it follows that $S \cdot \beta I \subseteq \beta I$, i.e. $\beta I$ is a closed $S$-invariant subset of $\beta S$. Thus, there is $M_{1}$, a minimal non-empty closed $S$-invariant subset of $\beta I$. It follows from Proposition 3.7 that $\left(M_{1}, S, \pi_{u}\right)$ is a universal minimal dynamical system for $S$, and so $M_{1}=\beta S \cdot p$ for any $p \in M_{1} \cdot M_{1}=\beta S \cdot p \supseteq \beta I \cdot p \supseteq M_{1} \cdot p=M_{1}$ for any $p \in M_{1}$. 
Since $M_{1}$ is $S$-invariant, it must also be $I$-invariant. Let $M_{2}$ be a minimal nonempty closed $I$-invariant subset of $M_{1}$. Then $\left(M_{2}, I, \pi_{u}\lceil I)\right.$ is a universal minimal dynamical system for $I$ by Proposition 3.7, and so $M_{2}=\beta I \cdot p$ for any $p \in M_{2}$. Thus, $M_{2}=\beta I \cdot p \supseteq M_{1} \cdot p=M_{1}$ for $p \in M_{2} \subseteq M_{1}$, and so $M_{1}=M_{2}$. It follows that $\left(M_{1}, I, \pi_{u} \mid I\right)$ is a universal minimal dynamical system for $I$.

(2) According to the proof of part (1), consider the universal minimal dynamical system $\left(M_{1}, S, \pi_{u}\right)$ for $S$ so that $M_{1} \subseteq \beta I$. Then $\left(M_{1}, I, \pi_{u}\lceil I)\right.$ is a universal minimal dynamical system for $I$. Thus there is an isomorphism $\phi:(M, I, \pi) \rightarrow$ $\left(M_{1}, I, \pi_{u} \backslash I\right)$.

For $s \in S$ and $x \in M$ define $\hat{\pi}(s)(x)=\phi^{-1}\left(\pi_{u}(s)(\phi(x))\right)$. Then $\hat{\pi}(s): M \rightarrow M$ is a continuous open mapping. It follows that $\hat{\pi}(s \cdot t)=\hat{\pi}(s) \circ \hat{\pi}(t)$ and so $(M, S, \hat{\pi})$ is a dynamical system. Since $\phi(\hat{\pi}(s)(x))=\phi\left(\phi^{-1}\left(\pi_{u}(s)(\phi(x))\right)\right)=\pi_{u}(s)(\phi(x))$ for any $s \in S$ and any $x \in M, \phi$ is an isomorphism from $(M, S, \hat{\pi})$ onto $\left(M_{1}, S, \pi_{u}\right)$. Thus $(M, S, \hat{\pi})$ is a universal minimal dynamical system for $S$.

Remark 3.11. Let $S=(S, \cdot)$ be a semigroup without the identity. Define $S^{1}=$ $S \cup\{1\}$ and define $1 \cdot s=s \cdot 1=s$ for any $s \in S$. Then $\left(S^{1}, \cdot\right)$ is a monoid. $S$ is a left ideal of $S^{1}$, and so by Theorem 3.5, if $\left(M, S^{1}, \pi\right)$ is a universal minimal dynamical system for $S^{1}$, then $(M, S, \pi\lceil S)$ is a universal minimal dynamical system for $S$. Thus we can restrict our investigation to universal minimal dynamical systems for monoids rather than semigroups.

\section{Syndetic Algebras of Sets}

In this section we shall deal with a certain family of Boolean algebras for a given semigroup $(S, \cdot)$. The family has the property that for any minimal dynamical system $(X, S)$ with a Boolean phase space the algebra $C O(X)$ is isomorphic to a member of this family, and vice versa. All algebras in the family are subalgebras of $\mathcal{P}(S)$, i.e. they are fields of sets. Our aim is to characterize $B(S)$ as such an algebra to give us a more explicit description of $B(S)$.

Definition 4.1. A set $A$ is called $S$-syndetic if $A \subseteq S$ and there is an integer $n \geq 0$ and $s_{0}, \ldots, s_{n} \in S$ so that $S=\bigcup_{i \leqslant n} l_{s_{i}}^{-1}[A]$.

Note that if $S$ is a group, the syndetic property reduces to covering $S$ by finitely many translations, i.e. $\bigcup_{i \leqslant n} s_{i} \cdot A=S$.

It is obvious that the class of all $S$-syndetic sets is closed under superset operation (and hence under union), and also under left translations from $S$, i.e. for every $s \in S$ and every $S$-syndetic set $A, l_{s}[A]=s \cdot A$ is again $S$-syndetic. However, this may not be true for $l_{s}^{-1}[A]$.

Definiton 4.2. Let $(X, S, \pi)$ be a dynamical system and let $x \in X$ and $U \subseteq X$. Then the set of returns of $x$ to $U$ is defined by $\operatorname{Ret}(x, U)=\{s \in S: \pi(s)(x) \in U\}$.

Lemma 4.3. Let $(X, S, \pi)$ be a dynamical system. Then

(1) For any open $U \subseteq X$ and any $x \in X, l_{s}^{-1}[\operatorname{Ret}(x, U)]=\operatorname{Ret}\left(x, \pi(s)^{-1}[U]\right)$.

(2) $(X, S, \pi)$ is minimal iff for any non-empty open $U \subseteq X$ and any $x \in X$, $\operatorname{Ret}(x, U)$ is an $S$-syndetic set.

Proof. (1) $l_{s}^{-1}[\operatorname{Ret}(x, U)]=\{t \in S: s \cdot t \in \operatorname{Ret}(x, U)\}=\{t \in S: \pi(s \cdot t)(x) \in U\}=$ $\{t \in S: \pi(s)(\pi(t)(x)) \in U\}=\left\{t \in S: \pi(t)(x) \in \pi(s)^{-1}[U]\right\}=\operatorname{Ret}\left(x, \pi(s)^{-1}[U]\right)$. 
(2) In accordance with our convention we shall drop the reference to $\pi$ for this part of the proof. Assume that $(X, S)$ is minimal. Let $U$ be a non-empty open subset of $X$. Then $\bigcup_{s \in S} s^{-1}[U]=X$. Due to compactness of $X$ there are finitely many $s_{0}, \ldots, s_{n} \in S$ so that $\bigcup_{i \leqslant n} s_{i}^{-1}[U]=X$. So for any $x \in X$ we have $\bigcup_{i \leqslant n} l_{s_{i}}^{-1}[\operatorname{Ret}(x, U)]=$ $S$, i.e. $\operatorname{Ret}(x, U)$ is $S$-syndetic.

On the other hand fix an $x \in X$ and an open non-empty $U \subseteq X$. As $\operatorname{Ret}(x, U)$ is $S$-syndetic, there are $s_{0}, \ldots, s_{n} \in S$ so that $\bigcup_{i \leqslant n} l_{s_{i}}^{-1}[\operatorname{Ret}(x, U)]=S$, so $S=$ $\operatorname{Ret}\left(x, \bigcup_{i \leqslant n} s_{i}^{-1}[U]\right)=\operatorname{Ret}\left(x, \bigcup_{s \in S} s^{-1}[U]\right)$. Thus, for any $x \in X$ and any non-empty open $U \subseteq X, \bigcup_{x \in X} \operatorname{Orb}(x) \subseteq \bigcup_{s \in S} s^{-1}[U]$. If $X \neq \bigcup_{s \in S} s^{-1}[U]$, then $Y=X-\bigcup_{s \in S} s^{-1}[U]$ is a non-empty closed $S$-invariant subset of $X$ and so $O r b(y) \cap \bigcup_{s \in S} s^{-1}[U]=\emptyset$ for any $y \in Y$, a contradiction. Thus, $X=\bigcup_{s \in S} s^{-1}[U]$ for any open non-empty $U \subseteq X$, and so $(X, S)$ is minimal.

The above observations motivate the following definition.

Definition 4.4. An algebra $\mathcal{A}$ of subsets of $S$ is called $S$-syndetic if every $A \in \mathcal{A}-\{\emptyset\}$ is $S$-syndetic, and if for every $s \in S$ and every $A \in \mathcal{A}, l_{s}^{-1}[A] \in \mathcal{A}$.

The set of all $S$-syndetic algebras is ordered by inclusion, therefore, by Zorn's principle, there always exist maximal ones. They play an important rôle in our investigation of universal minimal dynamical systems.

Theorem 4.5. For a semigroup $S, B(S)$ is isomorphic to a maximal $S$-syndetic algebra. In particular, for any $q \in M(S), B(S) \cong\{\operatorname{Ret}(q, U): U \in C O(M(S))\}$. If $S$ has an identity element, then every maximal $S$-syndetic algebra is isomorphic to $B(S)$ and thus a complete Boolean algebra.

Proof. Consider the universal minimal dynamical system $(M, S)$ as a subsystem of $(\beta S, S)$.

Fix a $q \in M$. According to Lemma 3.8, $R_{q}:(\beta S, S) \rightarrow(M, S)$ is a homomorphism onto $(M, S)$. Then $\rho_{q}: C O(M) \rightarrow \mathcal{P}(S)$ defined by $\rho_{q}\left(A^{*} \cap M\right)=\{s \in S:$ $\left.l_{s}^{-1}[A] \in q\right\}=\operatorname{Ret}_{(\beta S, S)}\left(q, A^{*}\right)=\operatorname{Ret}_{(M, S)}\left(q, A^{*} \cap M\right)$ for any $A \subseteq S$ is a Boolean homomorphism dual to $R_{q}$, and thus an embedding. It follows that $C O(M) \cong$ $r n g\left(\rho_{q}\right)$, where $r n g\left(\rho_{q}\right)$ denotes the range of $\rho_{q}$. From Lemma 4.3 it follows that not only $\rho_{q}\left(A^{*} \cap M\right)$ is an empty or $S$-syndetic set, but also $l_{s}^{-1}\left[\rho_{q}\left(A^{*} \cap M\right)\right]=$ $l_{s}^{-1}\left[\operatorname{Ret}\left(q, A^{*} \cap M\right)\right]=\operatorname{Ret}\left(q, s^{-1}\left[A^{*} \cap M\right)\right]=\rho_{q}\left(s^{-1}\left[A^{*} \cap M\right]\right) \in \operatorname{rng}\left(\rho_{q}\right)$. Thus, $\operatorname{rng}\left(\rho_{q}\right)$ is an $S$-syndetic algebra.

It remains to show that $\mathcal{A}=r n g\left(\rho_{q}\right)$ is a maximal $S$-syndetic algebra. Consider an $S$-syndetic algebra $\mathcal{B} \supseteq \mathcal{A}$. For any $s \in S$, let $h_{s}: \mathcal{P}(S) \rightarrow \mathcal{P}(S)$ be the Boolean homomorphism defined by $h_{s}(A)=l_{s}^{-1}[A]$ for any $A \subseteq S$. The Boolean dynamical $\operatorname{system}\left(\mathcal{B},\left\{h_{s}\lceil\mathcal{B}: s \in S\}\right)\right.$ is minimal and $\left(\mathcal{A},\left\{h_{s}\lceil\mathcal{A}: s \in S\}\right)\right.$ is universal minimal, and $i d_{\mathcal{A}}$ is a homomorphism from $\left(\mathcal{A},\left\{h_{s}\lceil\mathcal{A}: s \in S\}\right)\right.$ into $\left(\mathcal{B},\left\{h_{s} \mid \mathcal{B}: s \in S\right\}\right)$. From Lemma 3.8 it follows that $i d_{\mathcal{A}}$ is an isomorphism, giving $\mathcal{A}=\mathcal{B}$. 
In summary, whenever $q \in M$, then $B(S) \cong r n g\left(\rho_{q}\right)=\{\operatorname{Ret}(q, U): U \in$ $C O(M)\}$ that is a maximal $S$-syndetic algebra.

For the second part of the proof we assume that $S$ has the identity element $j$. Consider a maximal $S$-syndetic algebra $\mathcal{A}$. We shall find a $q \in M$ so that $\mathcal{A}=\{\operatorname{Ret}(q, U): U \in C O(M)\}$.

$\left(\operatorname{Ult}(\mathcal{A}),\left\{f_{s}: s \in S\right\}\right)$ is a minimal dynamical system, where $\operatorname{Ult}(\mathcal{A})$ is the Stone space of the Boolean algebra $\mathcal{A}$ and for each $s \in S, f_{s}$ is the mapping dual to $h_{s}\lceil\mathcal{A}$. As such it is a factor of $(M, S)$, i.e. there is a homomorphism $\phi:(M, S) \rightarrow(\operatorname{Ult}(\mathcal{A}), S)$ that is onto. Consider the ultrafilter $p \in \operatorname{Ult}(\mathcal{A})$ defined by $p=\{A \in \mathcal{A}: j \in A\}$. There is some $q \in M$ so that $\phi(q)=p$. If $A \in \mathcal{A}$, let $\hat{A}$ denote the set $\{p \in U l t(\mathcal{A}): A \in p\}$. Since $\phi(s(q))=f_{s}(\phi(q))=f_{s}(p)$, it follows that $\operatorname{Ret}(p, \hat{A})=\operatorname{Ret}\left(q, \phi^{-1}[\hat{A}]\right)$. Due to the selection of $p$ we have for any $A \in \mathcal{A}$ $\operatorname{Ret}(p, \hat{A})=\left\{s \in S: f_{s}(p) \in \hat{A}\right\}=\left\{s \in S: A \in f_{s}(p)\right\}=\left\{s \in S: l_{s}^{-1}[A] \in p\right\}=$ $\left\{s \in S: j \in l_{s}^{-1}[A]\right\}=\{s \in S: s \cdot j \in A\}=\{s \in S: s \in A\}=A$. Thus, $\mathcal{A}=$ $\{\operatorname{Ret}(p, \hat{A}): A \in \mathcal{A}\}=\left\{\operatorname{Ret}\left(q, \phi^{-1}[\hat{A}]\right): A \in \mathcal{A}\right\} \subseteq\{\operatorname{Ret}(q, U): U \in C O(M)\}$, as $\phi^{-1}[\hat{A}]$ is a clopen subset of $M$. From maximality of $\mathcal{A}$ it follows that $\mathcal{A}=$ $\{\operatorname{Ret}(q, U): U \in C O(M)\}$.

Proposition 4.6. Let $(M, S)$ be a universal minimal dynamical system for a monoid $S$ that is a subsystem of the ultrafilter dynamical system $(\beta S, S)$. Then there are at most as many distinct maximal $S$-syndetic algebras as there are idempotent ultrafilters in $M$.

Proof. We showed in section 3, paragraph (c) that $M=\bigcup_{e \in \operatorname{Id}(M)} e \cdot M$, and each $e \cdot M$ is a group and $e$ is its identity. Consider $p \in e \cdot M$ for an $e \in \operatorname{Id}(M) . R_{p}: M \rightarrow M$ is an automorphism of $(M, S)$ and $R_{p}(e)=p$. Take any $\operatorname{Ret}(p, U)$ for some $U \in C O(M)$. Since $e$ is an idempotent of $M, \operatorname{Ret}\left(e, R_{p}^{-1}[U]\right)=\{s \in S: s(e) \in$ $\left.R_{p}^{-1}[U]\right\}=\left\{s \in S: R_{p}(s(e)) \in U\right\}=\left\{s \in S: s\left(R_{p}(e)\right) \in U\right\}=\operatorname{Ret}(p, U)$. By Theorem 4.5, for a given maximal $S$-syndetic algebra $\mathcal{A}$ there is $q \in M$ so that $\mathcal{A}=\{\operatorname{Ret}(q, U): U \in C O(M)\}=\{\operatorname{Ret}(e, U): U \in C O(M)\}$, where $e$ is the unique idempotent of $M$ so that $q \in e \cdot M$. It follows that there are at most as many distinct maximal $S$-syndetic algebras as there are idempotents in $M$.

Remark 4.7. On the one hand, if $\mathcal{A}$ is an $S$-syndetic algebra, $(\operatorname{Ult}(\mathcal{A}), S)$ is a minimal dynamical system. On the other hand, if $(X, S)$ is a minimal dynamical system and $X$ is a Boolean space, then $C O(X)$ can be embedded into a maximal $S$ syndetic algebra and hence $C O(X)$ is isomorphic to an $S$-syndetic algebra. In this sense, $S$-syndetic algebras represent all minimal dynamical systems with Boolean phase spaces.

Example 4.8. Let $S$ be a left zero semigroup. Then $\mathcal{P}(S)$ is the only maximal $S$-syndetic algebra, and so $B(S) \cong \mathcal{P}(S)$. Let $S$ be in addition countable. Then, as in Example 3.4 (i), we can obtain a minimal dynamical system $\left(2^{\omega}, S\right)$ since $2^{\omega}$ has a dense countable subset. For any $x \in 2^{\omega},\left\{\operatorname{Ret}(x, U): U \in C O\left(2^{\omega}\right)\right\}$ is an $S$-syndetic algebra. Since it is isomorphic with $C O\left(2^{\omega}\right)$, it is not a regular subalgebra of $\mathcal{P}(S)$. This illustrates the fact that $S$-syndetic algebras do not have to be ordered by regular inclusion.

For a description of structural properties of maximal $S$-syndetic algebras it is important to know when an $S$-syndetic subalgebra of another $S$-syndetic algebra is 
its regular subalgebra. The following lemma (known for groups - see [A]) describes some of the conditions.

Lemma 4.9. Let $(X, S)$ and $(Y, S)$ be minimal dynamical systems for a semigroup $S$. Let $\phi:(X, S) \rightarrow(Y, S)$ be a homomorphism. If $S$ acts on $Y$ via semiopen mappings, then $\phi$ itself must be semiopen.

Proof. Take a non-empty open $U \subseteq X$. Take a non-empty open $V$ so that $\operatorname{cl}(V) \subseteq$ $U$, it is possible as $X$ is regular. Due to minimality of $(X, S)$, there are $s_{0}, \ldots, s_{n} \in$ $S$ so that $X=\bigcup_{i \leqslant n} s^{-1}[c l(V)]$. By Lemma 1.5 (1) $\phi$ must be onto $Y$, and so $Y=\phi[X]=\phi\left[\bigcup_{i \leqslant n} s_{i}^{-1}[c l(V)]\right]=\bigcup_{i \leqslant n} \phi\left[s_{i}^{-1}[c l(V)]\right] \subseteq \bigcup_{i \leqslant n} s_{i}^{-1}[\phi[c l(V)]] \subseteq Y$ as $\phi$ is a homomorphism. Since $\phi[\operatorname{cl}(V)]$ is closed, $s_{i}^{-1}[\phi[c l(V)]]$ must have a non-empty interior for some $i \leqslant n$. Since $s_{i}$ is semiopen, $\phi[c l(V)]$ must have a non-empty interior, and, consequently, $\phi[U]$ must have a non-empty interior.

Corollary 4.10. Let $S$ be a group, and let $\mathcal{A}$ and $\mathcal{B}$ be $S$-syndetic algebras. Whenever $\mathcal{A} \subseteq \mathcal{B}$, then $\mathcal{A}$ is a regular subalgebra of $\mathcal{B}$.

In the following we shall deal with the relation of the associated Boolean algebras for distinct semigroups utilizing the fact that in universal minimal dynamical systems semigroups act via open mappings.

Proposition 4.11. Let $S=(S, \cdot)$ and $T=(T, \cdot)$ be semigroups. Let $\phi:(S, \cdot) \rightarrow$ $(T, \cdot)$ be a (semigroup) homomorphism onto $(T, \cdot)$. Let $(M(S), S)$ and $(M(T), T)$ be universal minimal dynamical systems for respective semigroups. Then there is $\xi: M(S) \rightarrow M(T)$, a continuous open mapping onto $M(T)$ satisfying $\xi(s(p))=$ $\phi(s)(\xi(p))$ for any $s \in S$ and any $p \in M(S)$.

Proof. Let $\phi^{*}: \beta S \rightarrow \beta T$ be the continuous extension of $\phi$. Then $\phi^{*}$ satisfies $\phi^{*}(s \cdot p)=\phi(s) \cdot \phi^{*}(p)$ for any $s \in S$ and any $p \in \beta S$.

Consider a universal minimal dynamical system $(M(S), S)$ as a subsystem of the ultrafilter dynamical system. Set $M(T)=\phi^{*}[M(S)]$.

$M(T)$ is a $T$-invariant subset of $\beta T$, for if $t \in T$ and $p \in M(T)$, then for some $s \in S$ and $q \in M(S), \phi(s)=t$ and $\phi^{*}(q)=p$. It follows that $t \cdot p=\phi(s) \cdot \phi^{*}(q)=$ $\phi^{*}(s \cdot q) \in M(T)$.

$T$ acts minimally on $M(T)$, for if $p \in M(T)$, then for some $q \in M(S), \phi^{*}(q)=p$. Hence $\operatorname{Orb}_{(M(T), T)}(p)=\{t \cdot p: t \in T\}=\left\{\phi(s) \cdot \phi^{*}(q): s \in S\right\}=\left\{\phi^{*}(s \cdot q): s \in\right.$ $S\}=\phi^{*}\left[\operatorname{Orb}_{(M(S), S)}(q)\right]$. Since $(M(S), S)$ is minimal, $\operatorname{Orb}_{(M(S), S)}(q)$ is dense in $M(S)$, and, consequently, $\operatorname{Orb}_{(M(T), T)}(p)$ is dense in $M(T)$.

$(M(T), T)$ as a minimal dynamical subsystem of $(\beta T, T)$ must be universal for $T$ and so by Theorem $3.3 T$ acts on $M(T)$ via open mappings. By Lemma 4.9 the mapping $\xi=\phi^{*} \uparrow M(S)$ is semiopen. As $M(T)$ is extremally disconnected, $\xi$ must be open.

Corollary 4.12. If $S$ and $T$ are moreover monoids, then every maximal $T$-syndetic algebra is completely embeddable into any maximal $S$-syndetic algebra.

Remark 4.13. Recall the congruence of effectivness $\rho(S)$. For $s \in S$, let $[s]$ denote the class of all elements of $S$ in congruence $\rho(S)$ with $s$. Let $(M, S, \pi)$ be a universal minimal dynamical system. Let $\bar{S}=S / \rho(S)$ and let $\bar{\pi}: \bar{S} \rightarrow C(M, M)$ be defined 
by $\bar{\pi}[s]=[\pi(s)]$ for any $[s] \in \bar{S}$. It is clear that $(M, \bar{S}, \bar{\pi})$ is a minimal dynamical system for $\bar{S}$ and as such a factor of $(M(\bar{S}), \bar{S})$. On the other hand the canonical homomorphism $\phi: S \rightarrow \bar{S}$ is onto, and thus, by Proposition 4.11, $(M(\bar{S}), \bar{S})$ is a factor of $(M, \bar{S}, \bar{\pi})$. It follows that $(M, \bar{S}, \bar{\pi})$ is a universal minimal dynamical system for $\bar{S}$.

Moreover, if $\mathcal{A}$ is a maximal $S$-syndetic algebra, then if $A \in \mathcal{A}$ and $a \in A$, it follows that $[a] \subseteq A$. For $\mathcal{A}=\{\operatorname{Ret}(q, U): U \in C O(M)\}$ by Theorem 4.5 for some $q \in M$. Hence $A=\operatorname{Ret}(q, U)$ for some $U \in C O(M)$. If $a \in A$, then $\pi(a)(q) \in U$. If $b \in[a]$, then $\pi(b)=\pi(a)$ and so $\pi(b)(q) \in U$ as well. Hence $b \in \operatorname{Ret}(q, U)$, i.e. $b \in A$.

Since any $S$-syndetic algebra is included in a maximal one, the elements of all $S$-syndetic algebras are "saturated" with respect to $\rho(S)$.

\section{Structural properties of $B(S)$}

For any semigroup $S$ we have an extremally disconnected Hausdorff space $M(S)$ with a system of open continuous selfmaps of $M(S)$, or dually, a complete Boolean algebra $B(S)$ and a system $\left\{h_{s}: s \in S\right\}$ of complete autohomomorphisms of $B(S)$.

It is natural to ask how the size of $B(S)$ depends on $S$. In fact there is no simple relationship. We saw (Example 4.8) that for a left zero semigroup $S,|B(S)|=$ $|\mathcal{P}(S)|$ and an example (3.10) of $S$ for which $|B(S)|=|S|$. Later in this section we shall present properties of semigroups for which $B(S)$ is trivial, i.e. $B(S)=\{\mathbb{O}, \mathbb{I}\}$. We shall also discuss when $B(S)$ is atomic or atomless. We shall conclude the section by showing that a commutative semigroup $S$ acts in the universal minimal dynamical system via homeomorphisms and that $B(S)$ satisfies the c.c.c. It is known that if $G$ is a commutative group, then $|B(G)|=2^{|G|}$ ([T], $[\mathrm{CvM}]$ ). We are able to show that the Cohen algebra $\mathbb{C}\left(2^{|G|}\right)$ can be completely embedded into $B(G)$, provided $G$ is infinite. It is an open problem how much $B(G)$ differs from $\mathbb{C}\left(2^{|G|}\right)$ for an uncountable $G$. For countable $G$ see section 6 .

If $F$ is a cardinal function on Boolean algebras such that for any $u \in \mathcal{B}$, $F(\mathcal{B}\lceil u) \leq F(\mathcal{B})$, then $\mathcal{B}$ is said to be $F$-homogeneous if $F(\mathcal{B})=F(\mathcal{B}\lceil u)$ for any $u \in \mathcal{B}-\left\{\mathbb{O}_{\mathcal{B}}\right\}$, and weakly $F$-homogeneous if there is a partition $\left\{u_{i}: i \in I\right\}$ of $\mathbb{I}_{\mathcal{B}}$ such that each $\mathcal{B}\left\lceil u_{i}\right.$ is $F$-homogeneous and $F\left(\mathcal{B}\left\lceil u_{i}\right)=F\left(\mathcal{B}\left\lceil u_{j}\right)\right.\right.$ for $i, j \in I$. $\pi(\mathcal{B})=\min \left\{|H|: H \subseteq \mathcal{B}-\left\{\mathbb{O}_{\mathcal{B}}\right\} \& H\right.$ is dense in $\left.\mathcal{B}\right\}$, so-called density of $\mathcal{B}$.

Proposition 5.1. Let $S$ be an infinite semigroup. Then

(1) $B(S)$ satisfies the $|S|^{+}$-c.c.;

(2) $B(S)$ is weakly $\pi$-homogeneous.

Proof. (1) Follows from the fact that for any $x \in M, \operatorname{Orb}(x)$ is dense in $M$ and $|\operatorname{Orb}(x)| \leqslant|S|$.

(2) Let $B$ denote the algebra $B(S)$. Since the cardinal function $\pi$ is order preserving, there is a partition $P$ of $\mathbb{I}_{B}$ such that $B\lceil u$ is $\pi$-homogeneous for any $u \in P$. We want to show that $\pi(B \backslash u)=\pi(B \backslash v)$ for any $u, v \in P$. As $(M, S)$ is minimal, there is an $s \in S$ so that $h_{s}(u) \wedge v \neq \mathbb{O}_{B}$. Consider $g$ defined by $g(x)=h_{s}(x) \wedge v, x \in B$. Then $g: B \rightarrow B\lceil v$ is a complete homomorphism. Set $u_{1}=u-\bigvee \operatorname{Ker}(g)$. Then $\mathbb{O}_{B}<u_{1} \leqslant u$ and $g\left(u_{1}\right)=g(u)$. Set $v_{1}=g\left(u_{1}\right)$. Then $\mathbb{O}_{B} \neq v_{1} \leqslant v$ and $g: B\left\lceil u_{1} \rightarrow B\left\lceil v_{1}\right.\right.$ is a complete embedding. It follows that $\pi\left(B\lceil u)=\pi\left(B\left\lceil u_{1}\right) \leqslant \pi\left(B\left\lceil v_{1}\right)=\pi(B\lceil v)\right.\right.\right.$. From the symmetry of the argument we can conclude that $\pi(B\lceil u)=\pi(B\lceil v)$. 
Fact 5.2. If $S$ is a finite semigroup, then $B(S)$ is finite and therefore atomic.

We will show that for an infinite $S$ only two cases are possible; either $B(S)$ is atomic, i.e. $M(S)$ has a dense set of isolated points, or $B(S)$ is atomless, i.e. $M(S)$ has no isolated points. For $s \in S$ we shall denote as Fix $(s)$ the set of fixed-points of the left translation $l_{s}$, i.e. Fix $(s)=\{x \in S: s \cdot x=x\}$. For an infinite group $G$, Proposition 5.4 implies immediately that $B(G)$ is atomless as Fix $(g)=\emptyset$ for any $g \in G-\left\{1_{G}\right\}$.

The next lemma is well known (see e.g. [CN], Theorem 9.2).

Lemma 5.3. Consider the ultrafilter dynamical system $(\beta S, S)$. Let $p \in \beta S$. Then $s(p)=p$ iff $F i x(s) \in p$.

Proposition 5.4. Let $S$ be a semigroup. Then the following are equivalent:

(1) $M(S)$ has an isolated point;

(2) $M(S)$ has a dense subset $X$ of isolated points, and so $M(S)$ is homeomorphic to $\beta X$;

(3) $\mathcal{F}_{A}=\{F i x(s): s \in A\}$ is centred for some $S$-syndetic A (i.e. every finite set of elements of $\mathcal{F}_{A}$ has a non-empty intersection).

Proof. (1) $\rightarrow(2)$. Let $p$ be an isolated point of $M(S)$. Then $\{p\}$ is an open subset of $M(S)$. Since for every $s \in S, s: M(S) \rightarrow M(S)$ is open, then every point of the orbit of $p$ is isolated, and $\operatorname{Orb}(p)$ is dense in $M(S)$ due to minimality of $M(S)$.

$(2) \rightarrow(3)$. Consider $(M(S), S)$ as a subsystem of $(\beta S, S)$. Let $p \in M(S)$ be an isolated point. Set $A=\operatorname{Ret}(p,\{p\})=\{s \in S: s(p)=p\}$. If $s \in A$, then $s(p)=p$, and so by Lemma 5.3, Fix $(s) \in p$. Thus, $\{$ Fix $(s): s \in A\}$ is centred and $A$ is $S$-syndetic.

$(3) \rightarrow(1)$. Take an ultrafilter $p \in \beta S$ that extends the centred family $\{F i x(s)$ : $s \in A\}$. Let $U$ be an open subset of $\beta S$ containing $p$. Since $A \subseteq \operatorname{Ret}(p, U), \operatorname{Ret}(p, U)$ is $S$-syndetic, and thus $p$ is an almost-periodic point in $(\beta S, S)$. It follows that $M=\operatorname{cl}(\operatorname{Orb}(p))$ is a minimal $S$-invariant closed subset of $\beta S$ and $p \in M$ (see [F]). As $A$ is $S$-syndetic, there are $s_{0}, \ldots, s_{n} \in S$ so that $S=\bigcup_{i \leqslant n} l_{s_{i}}^{-1}[A]$. If $s \in S$, then for some $i \leqslant n, s_{i} \cdot s \in A$. It follows that $\left(s_{i} \cdot s\right)(p)=p$, yielding $s(p) \in s_{i}^{-1}[\{p\}]$. Consequently, $\operatorname{Orb}(p) \subseteq \bigcup_{i \leqslant n} s_{i}^{-1}[\{p\}]$, yielding $M=\operatorname{cl}(\operatorname{Orb}(p)) \subseteq \bigcup_{i \leqslant n} s_{i}^{-1}[\{p\}]$. For some $i \leqslant n, s_{i}^{-1}[\{p\}]$ must have a non-empty interior. Since $s_{i}$ is an open mapping, $\{p\}$ must be open, i.e. $p$ is isolated.

The following lemma deals with the problem when $B(S)$ is trivial, i.e. when $|M(S)|=1$.

Lemma 5.5. $|M(S)|=1$ iff $\{$ Fix $(s): s \in S\}$ is centred.

Proof. First assume that $M(S)=\{p\}$. Then for every $s \in S, s(p)=p$ and by Lemma $5.3\{F i x(s): s \in S\} \subseteq p$, and hence centred.

On the other hand if $\{F i x(s): s \in S\}$ is centered, then there is an ultrafilter $p \in \beta S$ extending $\{$ Fix $(s): s \in S\}$. As in the proof of Proposition 5.4, part $(3) \rightarrow(1)$, we can assume that $p \in M(S)$. Since for every $s \in S, F i x(s) \in p$, by Lemma 5.3, $s(p)=p$. Thus $\operatorname{Orb}(p)=\{p\}$, and, consequently, $M(S)=\{p\}$.

Example 5.6. Here we present some simple applications of Proposition 5.4 and Lemma 5.5. 
(i) Consider a semigroup $S$ that has a right zero $z$, i.e. $s \cdot z=z$ for every $s \in S$. Then $z \in F i x(s)$ for every $s \in S$ and so $\{F i x(s): s \in S\}$ is centred. By Lemma $5.5, B(S)=\{\mathbb{O}, \mathbb{I}\}$.

(ii) In this example we discuss idempotent semigroups (also called bands). A semigroup $S$ is idempotent if all its elements are idempotents. We can define a partial order on $S$ by $a \leqslant b$ iff $a=a b=b a$. Since $a b a \leqslant a$ for any $a, b \in S$, if $a$ is $\leqslant-$ minimal, then $a b a=a$ for any $b \in S$.

We shall deal with two extreme cases of idempotent semigroups, totally noncommutative and commutative ones.

(a) Consider a nowhere commutative semigroup $S$, i.e. a semigroup so that for any $a, b \in S, a b=b a$ implies $a=b . S$ is an idempotent semigroup, for $a a^{2}=a^{2} a$ and so $a=a^{2}$. Moreover every two elements are inverse, i.e. for any $a, b \in S, a=a b a$ and $b=b a b$. For $a a b a=a^{2} b a=a b a$ and $a b a a=a b a^{2}=a b a$, so $a a b a=a b a a$, giving $a=a b a$.

We show that $B(S)$ is atomic. Fix an arbitrary $a \in S$. Since $a \in F i x(b)$ for any $b \in a S,\{F i x(b): b \in a S\}$ is centred. Since $a S$ is $S$-syndetic, by Proposition $5.4(3), B(S)$ is atomic.

(b) Consider a commutative band $S$. For an $a \in S$ and $x \in S$, $a x=x$ iff $x \leqslant a$. Hence Fix $(a)=\{x \in S: x \leqslant a\}$. Since $a b \leqslant a$ and $a b \leqslant b$ for any $a, b \in S$, Fix $(a b) \subseteq$ Fix $(a)$ and Fix $(a b) \subseteq$ Fix $(b)$. Consequently, $\{$ Fix $(a): a \in S\}$ is centered, and thus by Lemma $5.5,|M(S)|=1$.

(c) Consider an arbitrary idempotent semigroup $S$. We show that if $S$ has a $\leqslant-$ minimal element, then $B(S)$ must be atomic, by reducing the problem to the case (a). $I=\{a \in S: a$ is minimal $\}$ is a left ideal of $S$, for if $a \in I$ and $s \in S$, sa must again be minimal: if $c \leqslant s a$, then $c=c s a=s a c$, so $a c=a c s a=a$. Hence $c=s a c=s a$. Then $B(S) \cong B(I)$ by Theorem 3.5. Since $I$ is nowhere commutative, by (a), $B(I)$ is atomic, and so $B(S)$ is atomic as well.

We still cannot resolve the general problem whether $B(S)$ is atomic for any idempotent semigroup.

In the following part of this section we shall deal with the problem of homogeneity and weak homogeneity of $B(S)$. For an infinite group $G, B(G)$ is always homogeneous (see Theorem 5.7). $B(S)$ is weakly homogeneous, provided $S$ is a semigroup with a minimal left ideal. We were not able to prove our conjecture that $B(S)$ is weakly homogeneous for an arbitrary semigroup. Let us remark that in general $B(S)$ cannot be homogeneous (see Example 5.11).

Theorem 5.7. (i) If $S$ has a minimal left ideal, then $B(S)$ is weakly homogeneous.

(ii) If $S$ is a left cancellative semigroup so that $B(S)$ is infinite, then $B(S)$ is homogeneous.

The proof of Theorem 5.7 will be done using the following lemmas.

Lemma 5.8. Let $(M, S)$ be a universal minimal dynamical system for $S$. If there exists a partition $\left\{M_{i}: i \in I\right\}$ of $M$ so that $(\forall x, y \in M)(\forall i \in I)(\forall s \in S)(x, y \in$ $\left.M_{i} \Longleftrightarrow s(x)=s(y)\right)$, then $B(S)$ is weakly homogeneous. If moreover $\left|M_{i}\right|=1$ for every $i \in I$ and $B(S)$ is infinite, then $B(S)$ is homogeneous.

Proof. The condition about the partition of $M$ says that for any $s, t \in S$, partitions $\left\{s^{-1}[\{x\}]: x \in M\right\}$ and $\left\{t^{-1}[\{y\}]: y \in M\right\}$ are the same. 
Our aim is to show that for any two distinct clopen non-empty subsets $U, V \subseteq M$ there is a homeomorphism $\phi: M \rightarrow M$ so that $\phi[U] \cap V \neq \emptyset$. We can assume that $U$ and $V$ are in fact disjoint.

We shall use the fact that for any clopen non-empty $W \subseteq M, \bigcup_{s \in S} s[W]$ is dense in $M$, as due to minimality of $(M, S)$ orbit of each $x \in W$ is dense in $M$.

There is $s \in S$ so that $U^{\prime}=s[M] \cap U \neq \emptyset$. It follows that $U^{\prime}$ is clopen. Denote $s^{-1}\left[U^{\prime}\right]$ as $W$. Then $W$ is a non-empty clopen subset of $S$. Moreover $W$ is saturated, i.e. if $M_{i} \cap W \neq \emptyset$, then $M_{i} \subseteq W$, for any $i \in I$.

There is $t \in S$ so that $V^{\prime}=t[W] \cap V \neq \emptyset$. It follows that $V^{\prime}$ is clopen. Set $W^{\prime}=t^{-1}\left[V^{\prime}\right]$. Then $W^{\prime}$ is a non-empty clopen subset of $W$ that is also saturated. The last step is to set $U^{\prime \prime}=s\left[W^{\prime}\right]$. $U^{\prime \prime}$ is a non-empty clopen subset of $U^{\prime}$. Since $s: M \rightarrow s[M]$ is a continuous map onto an extremally disconnected space $s[M]$, there exists a closed non-empty $M^{\prime} \subseteq M$ and an irreducible homeomorphism $\hat{s}: M^{\prime} \rightarrow s[M]$ so that $\hat{s}=s\left\lceil M^{\prime}\right.$ (see [En], 6.3.19 (c)). $M^{\prime}$ must be a selector with respect to the partition $\left\{s^{-1}[\{x\}]: x \in M\right\}$, and hence a selector with respect to the partition $\left\{M_{i}: i \in I\right\}$. Then $t \circ \hat{s}^{-1} \mid U^{\prime \prime}$ is a continuous one-to-one map from $U^{\prime \prime}$ onto $V^{\prime}$. This can easily be extended to the desired homeomorphism $\phi: M \rightarrow M$.

If all $M_{i}$ 's are singletons, i.e. if $S$ acts via one-to-one mappings, and $C O(M)$ is infinite, then $M$ has no isolated points (if $M$ had an isolated point, then by 5.4 it would have a dense subset of isolated points and, consequently, all points of $M$ would be isolated, which contradicts the compactness of $M$ ). According to the first part of this proof and Theorem 2.4, there is a clopen non-empty $V \subseteq M$ with the property that every clopen non-empty subset of $V$ is homeomorphic to $V$. From minimality of $(M, S)$ we have finitely many $s_{0}, \ldots, s_{n} \in S$ so that $\bigcup_{j \leqslant n} s_{j}^{-1}[V]=M$. As every $s_{j}$ is one-to-one, each $s_{j}^{-1}[V]$ has the property that every clopen nonempty subset of $s_{j}^{-1}[V]$ is homeomorphic to $s_{j}^{-1}[V]$, and thus $M$ has the property as well, i.e. $C O(M)$ is homogeneous.

Lemma 5.9. Let $S$ be a left cancellative semigroup so that $B(S)$ is infinite. Then $B(S)$ is homogeneous.

Proof. Since $S$ is left cancellative, for any $s \in S$, left translation on $S$ is one-to-one. It follows that for any $s \in S$, left translation on $\beta S$ is one-to-one. Consider the universal minimal dynamical system $(M(S), S)$ as a subsystem of $(\beta S, S)$. Then for every $s \in S, s: M(S) \rightarrow M(S)$ is one-to-one. According to Lemma 5.8, $B(S)$ is homogeneous.

Lemma 5.10. Let $S$ be a left simple semigroup. Then $B(S)$ is weakly homogeneous.

Proof. Let $s \in S$ and $x, y \in S$. Assume $l_{s}(x)=l_{s}(y)$. Let $A=\left\{t \in S: l_{t}(x)=\right.$ $\left.l_{t}(y)\right\}$. Then $A \supseteq S \cdot s$. Since $S$ is left simple, we get $S \cdot s \subseteq A \subseteq S=S \cdot s$. Thus, we have $l_{s}(x)=l_{s}(y) \Longleftrightarrow l_{t}(x)=l_{t}(y)$, for any $s, t \in S$ and any $x, y \in S$. We show that for any $p, q \in \beta S$ and any $s, t \in S, s \cdot p=s \cdot q$ iff $t \cdot p=t \cdot q$. Assume $s \cdot p=s \cdot q$. It suffices to prove that $t \cdot p \subseteq t \cdot q$. Take $B \subseteq S$ so that $B \in t \cdot p$. Then $l_{t}^{-1}[B] \in p$, therefore $l_{s}\left[l_{t}^{-1}[B]\right] \in s \cdot p=s \cdot q$. From this we have $l_{s}^{-1}\left[l_{s}\left[l_{t}^{-1}[B]\right]\right] \in q$. Since both $s$ and $t$ define the same partition of $S, l_{s}^{-1}\left[l_{s}\left[l_{t}^{-1}[B]\right]\right]=l_{t}^{-1}[B]$, and so $l_{t}^{-1}[B] \in q$, giving $B \in t \cdot q$. The same holds true for $p, q \in M(S)$. According to Lemma 5.8, $B(S)$ is weakly homogeneous. 
Example 5.11. To illustrate Lemma 5.10, consider a left group $S$ that is a special case of a left simple semigroup. Then $S \cong G \times E$ for some left zero semigroup $E$ and a group $G$ (Theorem 2.1). By Proposition 7.4, $B(S) \cong \prod_{e \in E} B(G)$. Since $G$ is a group, $B(G)$ is finite or homogeneous (by Lemma 5.9), and so $B(S)$ is weakly homogenous. Note that if $E$ is sufficiently big, then $B(S)$ cannot be homogeneous.

Proof of Theorem 5.7. (i) If $I$ is a minimal left ideal of $S$, then $I$ is a left simple semigroup. According to Lemma 5.10, $B(I)$ is weakly homogeneous, and according to Theorem $3.5 B(I) \cong B(S)$, hence $B(S)$ is weakly homogeneous.

(ii) follows from Lemma 5.9.

In the conclusion of this section we turn our attention to commutative semigroups. As it turns out, commutative semigroups act in their universal minimal dynamical systems via homeomorphisms, which does not have to be the case for minimal systems. Consider, for example, the symbolic dynamical system $\left(2^{\omega}, \sigma\right)$ where $\sigma$ is the shift to the left. It is a dynamical system for the semigroup $(\omega,+)$. Now, if $X \subseteq 2^{\omega}$ is an infinite minimal closed invariant subset, then $\sigma$ is not one-toone on $X$ (see $[F]$, page 158$)$.

Proposition 5.12. Let $S$ be a commutative semigroup and let $(M, S, \pi)$ be a universal minimal dynamical system. Then for any $s \in S, \pi(s): M \rightarrow M$ is a homeomorphism.

Proof. Consider $(M, S, \pi)$ as a subsystem of the ultrafilter dynamical system. Let $s, t \in S$ and $x \in M$. Since $S$ is commutative, we get $\pi(s)(\pi(t)(x))=s \cdot(t \cdot x)=$ $(s \cdot t) \cdot x=(t \cdot s) \cdot x=t \cdot(s \cdot x)=\pi(t)(\pi(s)(x))$. Hence $\pi(s): M \rightarrow M$ is an autohomomorphism of $(M, S, \pi)$ and by Lemma 3.8 an automorphism of $(M, S, \pi)$. It follows that $\pi(s)$ is a homeomorphism of $M$.

In the following we show that for a commutative semigroup $S, B(S)$ satisfies the c.c.c. The actual proof is presented for left amenable semigroups, for it greatly simplifies it, using the well-known fact that commutative semigroups are left amenable $([\mathrm{P}],[\mathrm{W}])$. We start with the definition of left amenable semigroups.

Definition 5.13. A semigroup $S$ is said to be left amenable if there is a finitely additive normed measure $\mu$ on $\mathcal{P}(S)$ so that for any $A \subseteq S$ and any $s \in S$, $\mu\left(l_{s}^{-1}[A]\right)=\mu(A)$.

Lemma 5.14. If a semigroup $S$ is left amenable, then $B(S)$ satisfies the c.c.c.

Proof. We know that $B(S)$ is isomorphic to a maximal $S$-syndetic algebra $\mathcal{A}$ (see Theorem 4.5). It suffices to show that there is a strictly positive finitely additive finite measure on $\mathcal{A}$. Consider the measure $\mu$ as guaranteed by left amenability of $S$. We shall show that $\mu(A)>0$ for any $S$-syndetic set $A$ : there are finitely many $s_{0}, \ldots, s_{n} \in S$ so that $S=\bigcup_{i \leqslant n} l_{s_{i}}^{-1}[A]$. Since $\mu(A)=\mu\left(l_{s_{i}}^{-1}[A]\right)$ for any $i \leqslant n$, $\mu(S) \leqslant(n+1) \mu(A)$. It follows that $\mu(A)>0$. Thus, $\mu\lceil\mathcal{A}$ is a strictly positive finitely additive finite measure on $\mathcal{A}$.

Let us remark that it is also known that a semigroup $S$ is left amenable iff for every minimal dynamical system $(X, S)$ there exists a Borel probability measure on $X$ that is $S$-invariant ([G]). 


\section{Structural properties of $B(S)$ For countable $S$}

For a countable semigroup we can often provide a more detailed description of the structure of $B(S)$.

Proposition 6.1. [Ko1]

(1) A Cohen algebra $\mathcal{B}$ is isomorphic to $\mathbb{C}(\pi(\mathcal{B}))$.

(2) A semi-Cohen algebra $\mathcal{B}$ so that $\pi(\mathcal{B}) \leqslant \aleph_{1}$ is Cohen.

(3) A complete $\pi$-homogeneus subalgebra of a Cohen algebra is semi-Cohen.

The next theorem summarizes the main cases when $B(S)$ is Cohen.

Theorem 6.2. Let $S$ be a countable semigroup so that $B(S)$ is atomless. Then $B(S)$ is Cohen, provided that $S$ is either left cancellative, or has a minimal left ideal, or is commutative.

We shall prove the theorem using several lemmas.

Lemma 6.3. Let $\left(\mathcal{B},\left\{h_{s}: s \in S\right\}\right)$ be a Boolean minimal dynamical system for a countable semigroup $S$ so that $\mathcal{B}$ is an atomless complete Boolean algebra and each $h_{s}$ is a complete homomorphism from $\mathcal{B}$ onto $\mathcal{B}$. Then $\mathcal{B}$ is a Cohen algebra.

Proof. Since each $h_{s}$ is a complete homomorphism, for each $s \in S$, and each $a \in \mathcal{B}$ there is the least $m(s, a) \in \mathcal{B}$ so that $h_{s}(m(s, a)) \geq a$. Moreover, $m(s, a \vee b)=$ $m(s, a) \vee m(s, b)$ and $m(s, a \wedge b)=m(s, a) \wedge m(s, b)$, since each $h_{s}$ is onto (see $2.5(2))$.

Define $\mathcal{S}$ by

$\mathcal{A} \in \mathcal{S}$ iff $\mathcal{A}$ is a countable subalgebra of $\mathcal{B}$ such that $h_{s}[\mathcal{A}] \subseteq \mathcal{A}$ and $m(s, a) \in \mathcal{A}$ for any $s \in S$ and any $a \in \mathcal{A}$.

(a) It is easy to see that $\mathcal{S}$ is closed.

(b) $\mathcal{S}$ is unbounded, for if $X \in[\mathcal{B}]^{\aleph_{0}}$, then set $\mathcal{A}_{0}=\langle X\rangle, \mathcal{A}_{n+1}=\left\langle\mathcal{A}_{n} \cup\left\{h_{s}(a)\right.\right.$ : $\left.\left.a \in \mathcal{A}_{n}, s \in S\right\} \cup\left\{m(s, a): a \in \mathcal{A}_{n}, s \in S\right\}\right\rangle$. Then $\mathcal{A}=\bigcup_{n \in \omega} \mathcal{A}_{n} \in \mathcal{S}$ and $X \subseteq \mathcal{A}$.

(c) If $\mathcal{A} \in \mathcal{S}$, then $\mathcal{A}$ is a regular subalgebra of $\mathcal{B}$. Since $\mathcal{A}$ contains $m(s, a)$ for every $a \in \mathcal{A}$ and every $s \in S, h_{s}\lceil\mathcal{A}: \mathcal{A} \rightarrow \mathcal{A}$ is a regular homomorphism, so its dual is a semiopen mapping (in fact its dual is an open mapping, see 2.5 (2)). By Lemma 4.9 (in Boolean form) $\mathcal{A}$ is a regular subalgebra of $\mathcal{B}$.

(d) If $\mathcal{A}_{1}, \mathcal{A}_{2} \in \mathcal{S}$, then $\left\langle\mathcal{A}_{1} \cup \mathcal{A}_{2}\right\rangle \in \mathcal{S}$. This follows directly from the fact $m(s, a \vee b)=m(s, a) \vee m(s, b)$ and $m(s, a \wedge b)=m(s, a) \wedge m(s, b)$, for any $a, b \in \mathcal{A}_{1} \cup \mathcal{A}_{2}$, and any $s \in S$.

The fact that $\mathcal{B}$ is c.c.c. and weakly $\pi$-homogeneous follows from the proof of Proposition 5.1. Since $\mathcal{B}$ is atomless, it must be $\pi$-homogeneous. Thus $\mathcal{B}$ is a Cohen algebra.

Lemma 6.4. Let $S$ be a countable semigroup and let $(M, S)$ be a universal minimal dynamical system for $S$. If there exists a partition $\left\{M_{i}: i \in I\right\}$ of $M$ so that $(\forall x, y \in M)(\forall i \in I)(\forall s \in S)\left(x, y \in M_{i} \Longleftrightarrow s(x)=s(y)\right)$ and if $B(S)$ is atomless, then $B(S)$ is a Cohen algebra.

Proof. We know that $C O(M)$ is an atomless, weakly $\pi$-homogeneous, c.c.c. complete Boolean algebra (see 5.1), hence $\pi$-homogeneous.

For each $s \in S$, set $V_{s}=s[M]$. Define $\operatorname{SatCO}(M)=\{U \in C O(M):(\forall i \in$ $\left.I)\left(M_{i} \cap U \neq \emptyset \rightarrow M_{i} \subseteq U\right)\right\}$. It is straightforward to verify that 
(i) for every $s \in S, \operatorname{Sat} C O(M) \cong C O\left(V_{s}\right)$, and so $\operatorname{Sat} C O(M)$ is an atomless, $\pi$-homogeneous, c.c.c. complete Boolean algebra.

(ii) if $U \in C O(M)$, then $\widehat{U}=\bigcup_{M_{i} \cap U \neq \emptyset} M_{i} \in \operatorname{SatCO}(M)$, for $\widehat{U}=s^{-1}[s[U]]$.

(iii) for each $s \in S$, consider the complete homomorphism $h_{s}: C O(M) \rightarrow$ $C O(M)$ defined by $h_{s}(A)=s^{-1}[A], A \in C O(M)$. Then $h_{s}\lceil\operatorname{Sat} C O(M)$ : $\operatorname{SatCO}(M) \rightarrow \operatorname{SatCO}(M)$ is a complete homomorphism onto $\operatorname{SatCO}(M)$, since $\operatorname{SatCO}(M)$ is a complete subalgebra of $C O(M)$, and since if $U \in$ $\operatorname{SatCO}(M)$, then $s[U] \in C O(M)$ and $\widehat{s[U]} \in \operatorname{SatCO}(M)$ and $h_{s}(\widehat{s[U]})=$ $s^{-1}[\widehat{s[U]}]=U$.

(iv) ( $\operatorname{Sat} C O(M),\left\{h_{s}: s \in S\right\}$ ) is a minimal dynamical system for $S$.

According to Lemma 6.3, $\operatorname{Sat} C O(M)$ is Cohen. Thus, each $C O\left(V_{s}\right)$ is Cohen as well. Since $\mathbb{I}_{C O(M)}=\bigvee_{s \in S} V_{s}$ and $C O(M)$ satisfies the c.c.c., $C O(M)$ is Cohen as well.

Lemma 6.5. Let $S$ be a left simple countable semigroup so that $B(S)$ is atomless. Then $B(S)$ is Cohen.

Proof. As in the proof of Lemma 5.10, $s(x)=s(y) \Longleftrightarrow t(x)=t(y)$, for any $s, t \in S$ and any $x, y \in M(S)$. According to Lemma $6.4, B(S)$ is Cohen.

Proof of Theorem 6.2. (1) Let $S$ be left cancellative. As in the proof of Lemma 5.9, for every $s \in S, s: M(S) \rightarrow M(S)$ is one-to-one. According to Lemma 6.4, $B(S)$ is Cohen.

(2) Let $S$ have a minimal left ideal $I$. Then $I$ is a left simple countable semigroup, so by Lemma $6.5, B(I)$ is Cohen. By Theorem $3.5, B(S) \cong B(I)$.

(3) Let $S$ be a countable commutative semigroup. By Proposition 5.12, $S$ acts via homeomorphisms in the universal minimal dynamical system. Hence Lemma 6.4 applies.

We can summarize the structural properties of associated Boolean algebras for countable semigroups into the following theorem.

\section{Theorem 6.6.}

(1) For every countable semigroup $S, B(S)$ can be completely embedded into $\mathbb{C}\left(2^{\omega}\right)$, and thus it is an atomic or a semi-Cohen algebra.

(2) $[\mathrm{BB}]$ For every countable group $G, B(G) \cong \mathbb{C}(\pi(B(G)))$.

(3) $[\mathrm{T}]$ For every countable commutative group $G, B(G) \cong \mathbb{C}\left(2^{\omega}\right)$;

Proof. (1) Let $(F, \cdot)$ be a free semigroup with countably generators. $F$ is left cancellative, therefore $B(F) \cong \mathbb{C}(\pi(B(F)))$, by Theorem 6.2. Since $\pi(B(F))=2^{\omega}$ (see $[\mathrm{BB}]), B(F) \cong \mathbb{C}\left(2^{\omega}\right)$. There is a semigroup homomorphism $\phi$ from $F$ onto $S$, so by Proposition $4.11, B(S)$ can be completely embedded into $B(F)$.

(2) Follows from the fact that groups are left cancellative and that $B(G)$ is atomless, as $G$ is infinite. $G$.

(3) In [T] Turek showed that $\pi(B(G))=2^{|G|}$ for any infinite commutative group

Corollary 6.7. Assuming $C H$, for every countable semigroup $S$, either $B(S) \cong$ $\mathcal{P}(X)$ for some $|X| \leqslant \omega$, or $B(S) \cong \mathbb{C}(\pi(B(S)))$. 
Proof. Under $\mathrm{CH}, \pi(B(S)) \leqslant|B(S)| \leqslant \omega_{1}$. By Theorem 6.6 together with 6.1 (3), $B(S)$ is semi-Cohen, and so by $6.1(2)$ Cohen.

As a concluding remark we present the following problem: is it possible for a countable semigroup $S$ such that $B(S)$ is atomless that $\pi(B(S))$ is less than continuum?

\section{Group Reflections of Semigroups}

The category of groups is an algebraic and a reflective subcategory of the category of semigroups (see [HS]). It means that every semigroup $S$ has a group reflection, i.e. a unique (up to isomorphism) group $G(S)$ together with a homomorphism $\varphi: S \rightarrow G(S)$ so that if $H$ is an arbitrary group and $\phi: S \rightarrow H$ a homomorphism, then there exists a unique group homomorphism $\xi: G(S) \rightarrow H$ so that $\xi \circ \varphi=\phi$. It follows that $G(S)$ is generated by $\varphi[S]$. In [L] an explicit construction of the group reflection $G(S)$ of a commutative monoid $S$ is given (so-called Grothendieck group of $S$ ). In the introduction of this paper an example was given showing that in general the universal minimal dynamical system for $S$ and the universal minimal dynamical system for $G(S)$ need not necessarily be the same.

Why are we interested in group reflections of semigroups in the context of dynamical systems? We are going to present a class of semigroups having the property that the universal minimal dynamical system for $S$ and the universal minimal dynamical system for $G(S)$ are the same (we shall define later what we mean by this imprecise term 'same'), provided $S$ is from that class. For the lack of a better term we call it the class of group-like semigroups. The important aspect of this class is that it includes the important class of all commutative semigroups as well as the class of inverse semigroups, and the class of right groups. There may be other classes of semigroups that are included in the class of group-like semigroups as well. We conclude this section with a discussion of left groups even though they do not belong to the class of group-like semigroups, for if $S$ is a left group, then $B(S) \cong \prod_{e \in E} B(G)$ for some set $E$ and some group $G$.

We say that a semigroup $S$ is group-like if

(i) it acts via homeomorphisms in its universal minimal dynamical system, and

(ii) for an arbitrary group $H$ and an arbitrary homomorphism $\xi: S \rightarrow H$ so that $\xi[S]$ generates $H$, whenever a dynamical system $(X, H, \rho)$ is minimal for $H$, then $(X, S, \rho \circ \xi)$ is a minimal dynamical system for $S$.

Consider a group-like semigroup $S$. Let $(M, S, \pi)$ be a universal minimal dynamical system for $S$, then $\pi[S] \subseteq \operatorname{Hom}(M)$. Denote as $G(S)$ the subgroup of $\operatorname{Hom}(M)$ generated by $\pi[S]$. It is clear that $G(S)$ is unique up to isomorphism and so we are justified in denoting it by $G(S)$. The next lemma says that $G(S)$ is the group reflection of a group-like semigroup $S$.

Lemma 7.1. Let $S$ be a group-like semigroup. Let $(M, S, \pi)$ be a universal minimal dynamical system for $S$. Let $\xi: S \rightarrow H, H$ a group, be a homomorphism so that $\xi[S]$ generates $H$. Then there is a unique homomorphism $\psi$ from $G(S)$ onto $H$ so that $\psi \circ \pi=\xi$.

Proof. Consider a universal minimal dynamical system $(X, H, \rho)$. Since $S$ is grouplike, $(X, S, \rho \circ \xi)$ is minimal. Thus, there is a homomorphism $\sigma$ from $(M, S, \pi)$ onto $(X, S, \rho \circ \xi)$. It follows that if $\pi(s)=\pi(t)$, then $\xi(s)=\xi(t)$ for any $s, t \in S$, 
since $\rho$ is one-to-one as $H$, being a group, acts effectively. Thus, if we define $\psi(\pi(s))=\xi(s)$ for all $s \in S$, then $\psi$ is a well defined unique homomorphism from $\pi[S]$ onto $\xi[S]$ such that $\psi \circ \pi=\xi$. Since $\pi[S]$ generates $G(S)$ and $H$ is generated by $\psi[S], \psi: \pi[S] \rightarrow \psi[S]$ has a unique extension $\psi: G(S) \rightarrow H$.

The next lemma shows how the universal minimal dynamical systems for a grouplike $S$ and its group reflection $G(S)$ are related. That is a precise formulation of the statement in the introduction of this section, that they have 'the same' universal minimal dynamical systems. Note, that it implies that $B(S)$ and $B(G(S))$ are isomorphic.

Lemma 7.2. Let $S$ be a group-like semigroup. Let $(M, S, \pi)$ be a universal minimal dynamical system for $S$. Let $(X, G(S), \rho)$ be a universal minimal dynamical system for $G(S)$. Then $(X, S, \rho \circ \pi)$ is a universal minimal dynamical system for $S$.

Proof. Since $(M, S, \pi)$ is a universal minimal dynamical system for $S,(M, G(S), i d)$ is a minimal dynamical system for $G(S)$, where $i d$ is the identity function on $G(S)$. There is a homomorphism $\chi:(X, G(S), \rho) \rightarrow(M, G(S), i d)$ as $(X, G(S), \rho)$ is universal. Then $\chi:(X, \pi[S], \rho\lceil\pi[S]) \rightarrow(M, \pi[S], i d \mid \pi[S])$ is a homomorphism. It follows that $\chi:(X, S, \rho \circ \pi) \rightarrow(M, S, \pi)$ is a homomorphism. Since $S$ is group-like, $(X, S, \rho \circ \pi)$ is minimal. Thus, $(X, S, \rho \circ \pi)$ is a universal minimal dynamical system for $S$.

\section{(a) Commutative semigroups.}

Lemma 7.3. Let $S$ be a commutative semigroup, then $S$ is group-like.

Proof. First a little remark: consider a dynamical system $(X, S)$. For any $s \in S, s$ is a homomorphism of $(X, S)$, for $s(t(x))=(s t)(x)=(t s)(x)=t(s(x))$ (as $S$ is commutative) for any $t \in S$ and any $x \in X$. It follows that if $A$ is a minimal closed non-empty $S$-invariant subset of $X$, then $s[A]=A$.

We proved that $S$ acts via homeomorphisms in its universal minimal dynamical system in Proposition 5.12. Consider a group $H$ and a homomorphism $\xi: S \rightarrow H$ so that $\xi[S]$ generates $H$. Let $(X, H, \rho)$ be a minimal dynamical system for $H$. Our aim is to show that $(X, S, \rho \circ \xi)$ is minimal. Let $A$ be a minimal closed non-empty $S$-invariant subset of $X$. Denote by $\phi$ the mapping $\rho \circ \xi$. Then $\phi(s)[A]=A$ for any $s \in S$ (according to the remark at the beginning of this proof). Since $\phi(s)$ is a homeomorphism, $\phi(s)^{-1}[A]=A$ for any $s \in S$. Let $h \in H$. Since $\xi[S]$ generates $H$ and $S$ is commutative, there are $s, t \in S$ so that $h=\xi(s) \cdot \xi(t)^{-1}$, where $\xi(t)^{-1}$ denotes the inverse of $\xi(t)$ in $H$. Then $\rho(h)=\rho(\xi(s)) \circ \rho(\xi(t))^{-1}$. Hence $\rho(h)[A]=$ $A$. Thus, $A$ is a closed non-empty $H$-invariant subset of $X$. By the minimality of $(X, H, \rho), A=X$. So $(X, S, \rho \circ \xi)$ is minimal.

If $S$ is a commutative semigroup, then $G(S)$ is a commutative group (as it is generated by $\pi[S]$ ), and by Lemma $7.1, G(S)$ is the Grothendieck group of $S$ as it is the group reflection of $S$. By Lemma 7.2, $B(S)$ and $B(G(S))$ are isomorphic.

We can illustrate the above on the minimal dynamical system $(X, f)$ for the additive group of integers $(\mathbb{Z},+)$, where $f: X \rightarrow X$ is a single homeomorphism. Then $(X, f)$ is a minimal dynamical system for $(\omega,+)$. So for any $x \in X$, the positive orbit of $x=\left\{f^{n}(x): n \in \omega\right\}$ is dense in $X$. Moreover $B(\omega,+)=$ $B(\mathbb{Z},+)=\mathbb{C}\left(2^{\omega}\right)$. 
(b) Inverse semigroups. Let $S$ be an inverse semigroup (see section 2(a)). We shall denote by $\bar{a}$ the unique inverse of $a \in S$, i.e. $a=a \bar{a} a$ and $\bar{a}=\bar{a} a \bar{a}$.

If $S$ is an inverse semigroup, then $S$ can be represented by $\left\{\rho_{a}: a \in S\right\}$ where each $\rho_{a}$ is a partial left translation of $S$ and a bijection from $\bar{a} S$ onto $S a$. For $a, b \in S, \rho_{a \cdot b}=\rho_{a} \circ \rho_{b}$ and $\rho_{\bar{a}}=\rho_{a}^{-1}$ (see [CP], Theorem 1.20). Several properties of $S$ can be observed directly from this representation:

(i) for any $a \in S, \bar{a} a$ and $a \bar{a}$ are idempotents;

(ii) for any $a \in S, \bar{a}=a$ iff $a$ is an idempotent;

(iii) if $a, b$ are idempotents, so is $a b$ and $a b=b a$;

(iv) $\overline{a b}=\bar{b} \bar{a}$ and $\overline{\bar{a}}=a$;

(v) the set of right ideals $\{a S: a \in S\}$ is centred.

Now consider the ultrafilter dynamical system $(\beta S, S)$. The set $U=\bigcap_{a \in S}(a S)^{*}$ is a non-empty closed $S$-invariant subset of $\beta S$. Take $M$, a minimal $S$-invariant closed subset of $U$, then $(M, S)$ is a universal minimal dynamical system for $S$. We shall show that $S$ is group-like using this representation of the universal minimal dynamical system for $S$.

(vi) It is straightforward to show that $U=\bigcap_{e \in \operatorname{Id}(S)}(e S)^{*}=\bigcap_{b \in S}(a b S)^{*}$, for any $a \in S$

(vii) Let $T_{a}=L_{a} \uparrow U, a \in S, L_{a}$ the left translation on $\beta S$. Then $T_{a}: U \rightarrow U$ is a homeomorphism.

Given $q \in U$, set $\mathcal{C}=\left\{l_{a}^{-1}[A]: A \in q\right\} \cup\{b S: b \in S\}$. Then $\mathcal{C}$ is centered, for $l_{a}^{-1}[a b S] \supseteq b S$ for any $b \in S$. Let $p \in \beta S$ extend $\mathcal{C}$, since $b S \in p$ for any $b \in S, p \in U$. Furthemore, $A \in q \Rightarrow l_{a}^{-1}[A] \in \mathcal{C} \Rightarrow l_{a}^{-1}[A] \in p \Rightarrow A \in L_{a}(p)$. Hence $q \subseteq L_{a}(p)$, but since both are ultrafilters, $q=L_{a}(p)$. Hence $T_{a}$ is onto.

Consider $p \neq q \in U . p, q \in(a S)^{*}$, so there are disjoint sets $X, Y \subseteq a S$ so that $X \in p$ and $Y \in q . l_{a}^{-1}[X]=\rho_{\bar{a}}[X]$ and $l_{a}^{-1}[Y]=\rho_{\bar{a}}[Y]$, and so $l_{a}^{-1}[X]$ and $l_{a}^{-1}[Y]$ are disjoint as well as $\rho_{a^{-1}}$ being a bijection. $l_{a}^{-1}[X] \in L_{a}(p)$ and $l_{a}^{-1}[Y] \in L_{a}(q)$, so $L_{a}(p) \neq L_{a}(q)$. Thus, $T_{a}$ is one-to-one.

Now we are ready to show that $S$ is group-like. $S$ acts on $U$ via homeomorphisms (vii), and hence on $M$ as well. Let $H$ be a group and let $\xi: S \rightarrow H$ be a homomorphism so that $\xi[S]$ generates $H$. Let $(X, H, \rho)$ be a minimal dynamical system for $H$. Consider the dynamical system $(X, S, \rho \circ \xi)$. We are to prove that it is minimal. Let $A$ be an $S$-invariant (with respect to $(X, S, \rho \circ \xi)$ ) closed non-empty subset of $X$. Since for any $s \in S, s=s \bar{s} s, \xi(s)=\xi(s) \cdot \xi(\bar{s}) \cdot \xi(s)$, hence $1_{H}=\xi(\bar{s}) \cdot \xi(s)$, and so $\xi(s)^{-1}=\xi(\bar{s})$, where $\xi(s)^{-1}$ denotes the inverse element of $\xi(s)$ in the group $H$. Thus, $\rho(\xi(s))^{-1}[A]=\rho(\xi(\bar{s}))[A] \subseteq A$ and $\rho(\xi(s))[A] \subseteq A$ for any $s \in S$. Since $\xi[S]$ generates $H, A$ is $H$-invariant (with respect to $(X, H, \rho)$ ) and so $A=X$. Thus, $(X, S, \rho \circ \xi)$ is minimal.

For an inverse semigroup $S, G(S)$ is its group reflection (by Lemma 7.1), and $B(S)$ and $B(G(S))$ are isomorphic (by Lemma 7.2).

(c) Right groups. Let $S$ be a right group, i.e. a right simple and left cancellative semigroup. Then by Theorem 2.1 (in dual form) there are a group $G$ and a right zero semigroup $E$ so that $S \cong G \times E$. Observe that $S$ is group-like and that $G(S)=G$ : 
(1) $S$ acts via homeomorphisms in its universal minimal dynamical system.

Let $(M, S, \pi)$ be a universal minimal dynamical system for $S$. Let $e \in E$. Since $G \times\{e\}$ is a left ideal of $S$, by Theorem 3.5, $(M, G \times\{e\}, \pi \mid G \times\{e\})$ is a universal minimal dynamical system for $G \times\{e\}$. Since $G \times\{e\}$ is a group, each $\pi(g, e)$ for any $g \in G$ is a homeomorphism from $M$ onto $M$.

(2) Let $H$ be a group, and let $\xi: S \rightarrow H$ be a homomorphism so that $\xi[S]$ generates $H$. Let $(X, H, \rho)$ be a minimal dynamical system for $H$. Then $(X, S, \rho \circ \xi)$ is a minimal dynamical system for $S$.

Let $A$ be an $S$-invariant closed non-empty subset of $X$. Let $\phi=\rho \circ \xi$. Then $\left(\phi\left(g_{1}, e_{1}\right) \circ \phi\left(g_{1}^{-1} g_{2}, e_{2}\right)\right)[A]=\phi\left(g_{2}, e_{2}\right)[A]$ for any $\left(g_{1}, e_{1}\right),\left(g_{2}, e_{2}\right) \in G \times E$, where $g_{1}^{-1}$ denotes the inverse of $g_{1}$ in the group $G$. At the same time, $\left(\phi\left(g_{1}, e_{1}\right) \circ \phi\left(g_{1}^{-1} g_{2}, e_{2}\right)\right)[A] \subseteq \phi\left(g_{1}, e_{1}\right)[A]$. Hence $\left(\phi\left(g_{1}, e_{1}\right) \circ \phi\left(g_{1}^{-1} g_{2}, e_{2}\right)\right)[A]$ $\subseteq \phi\left(g_{1}, e_{1}\right)[A] \cap \phi\left(g_{2}, e_{2}\right)[A]$. It follows that $\{\phi(s)[A]: s \in S\}$ satisfies the finite intersection property. Since $X$ is compact, $B=\bigcap_{s \in S} \phi(s)[A]$ is a nonempty closed subset of $A$.

$$
\begin{aligned}
\phi\left(g_{1}, e_{1}\right)[B] & =\bigcap_{g \in G, e \in E}\left(\phi\left(g_{1}, e_{1}\right) \circ \phi(g, e)\right)[A] \\
& =\bigcap_{g \in G, e \in E} \phi\left(g_{1} g, e\right)[A]=B .
\end{aligned}
$$

Thus $B$ is $H$-invariant (with respect to $(X, H, \rho)$ ), and by the minimality of $(X, H, \rho), B=X$. It follows that $A=X$.

According to Lemma 7.1, $G$ is the group reflection of $S$, and by Lemma 7.2, $B(S)$ and $B(G)$ are isomorphic.

(d) Left groups. Although left groups do not belong to the class of group-like semigroups, we present them here since $B(S)$ for a left group $S$ is determined by a certain group. Recall that, by Theorem 2.1, there are a group $G$ and a left zero semigroup $E$ so that $S \cong G \times E$. $B(E) \cong \mathcal{P}(E)$ by Example 3.10. According to the next proposition, $B(S) \cong B(G) \otimes B(E) \cong \prod_{e \in E} B(G)$, where $\otimes$ denotes the free product in the category of complete Boolean algebras, i.e. it is the completion of $\left(B(G)-\left\{\mathbb{O}_{B(G)}\right\}\right) \times\left(B(E)-\left\{\mathbb{O}_{B(E)}\right\}\right)$.

Proposition 7.4. Let $S$ be a monoid and $E$ a left zero semigroup. Then $B(S \times E) \cong B(S) \otimes B(E)$.

Proof. We can assume that $B(S)$ is a maximal $S$-syndetic algebra and $B(E)=$ $\mathcal{P}(E)$. Some notation first.

If $A \subseteq S \times E$, then $\operatorname{pr}_{E}(A)$ and $p r_{S}(A)$ denote the projection of $A$ on $E$ or on $S$ respectively. $A_{e}$ denotes $\operatorname{pr}_{S}(A \cap S \times\{e\})$.

Note that if $\emptyset \neq B \subseteq E$, then $B$ is $E$-syndetic, and $A \times B$ is $S \times E$-syndetic iff $A$ is $S$-syndetic.

Define $\mathcal{A}=\emptyset \cup\left\{\bigcup_{i \leqslant n} A_{i} \times B_{i}: n \geq 1, A_{i} \neq \emptyset, B_{i} \neq \emptyset, A_{i} \in B(S), B_{i} \subseteq E\right\}$. Then $\mathcal{A}$ is an $S \times E$-syndetic syndetic algebra.

Let $\mathcal{C}$ be a maximal $S \times E$-syndetic algebra extending $\mathcal{A}$. Define $\mathcal{B}=\{A$ : $A \times E \in \mathcal{C}\}$. Then $\mathcal{B}$ is an $S$-syndetic alegbra and $B(S) \subseteq \mathcal{B}$. By maximality of $B(S), B(S)=\mathcal{B}$. 
Now take any non-empty $X \in \mathcal{C}$. Let $e \in \operatorname{pr}_{E}(X)$, and let $s \in S$. Since $l_{(s, e)}^{-1}[X]=l_{s}^{-1}\left[X_{e}\right] \times E$, so $l_{s}^{-1}\left[X_{e}\right] \in B(S)$. In particular, if $s=1_{S}$, then $X_{e} \in B(S)$, so $X_{e} \times\{e\} \in \mathcal{A}$. Since $X_{e} \times\{e\} \subseteq X, \mathcal{A}$ is dense in $\mathcal{C}$. Thus, $\mathcal{C} \cong B(S) \otimes B(E)$.

Remark 7.5. Let us remark that generally for two semigroups $S_{1}, S_{2}$, the universal minimal dynamical system for the product $S_{1} \times S_{2}$, i.e. $\left(M\left(S_{1} \times S_{2}\right), S_{1} \times S_{2}, \pi\right)$, is not isomorphic to the Gleason resolvent of the product $\left(M\left(S_{1}\right), S_{1}, \pi_{1}\right) \times$ $\left(M\left(S_{2}\right), S_{2}, \pi_{2}\right)$.

\section{Miscellaneous Results}

Let $S$ be a semigroup. Consider $(M(S), S)$ as a subsystem of the ultrafilter dynamical system. $M(S)$ is a left simple right cancellative subsemigroup of $(\beta S, \cdot)$ and has the structure of $G \times I d(M(S))$ for some group $G$. Natural questions about this structure include questions when the structure is "extreme" in some way, in particular when $|\operatorname{Id}(M(S))|=1$ or $|G|=1$.

It is possible that $|M(S)|=1$ (see Lemma 5.5). The next proposition shows that if $M(S)$ is infinite, then the answer to the first question is negative, i.e. $|\operatorname{Id}(M(S))|>1$. The proposition follows from deep results concerning nonhomogeneity of $F$-spaces by Frolík (see e.g. [BS]).

Proposition 8.1. Let $S$ be a semigroup. Let $(M(S), S)$ be a subsystem of the ultrafilter dynamical system. If $M(S)$ is infinite, then $|\operatorname{Id}(M(S))| \geq 2^{2^{\omega}}$.

Proof. As $M(S)$ is an infinite Hausdorff space it does include a countable discrete subset $X$. Since $M(S)$ is extremally disconnected, $\beta X$ can be embedded in $M(S)$ (for details see $[\mathrm{BS}]$ ). From the proof of Frolík's theorem that $F$-spaces are not homogeneous it follows that $\beta X$ has at least $2^{2^{\omega}}$ classes of non-homogeneity. Thus, there are at least $2^{2^{\omega}}$ points in $M(S)$ that cannot be mapped onto each other by a homeomorphism from $M(S)$ onto $M(S)$. Since for any $e \in \operatorname{Id}(M(S))$, every two elements of $e \cdot M(S)$ can be mapped onto each other by an automorphism, $I d(M(S)) \mid \geq 2^{2^{\omega}}$.

The other "extreme" is the case when $|G|=1$. The following proposition shows that it is intimately tied to the notion of proximality. If $(X, S)$ is a dynamical system, two points $x, y \in X$ are said to be proximal if for every open cover of $X$ there are an open set $U$ from the cover and an $s \in S$ so that both $s(x)$ and $s(y)$ fall in $U$. The system $(X, S)$ is called proximal if all pairs of points of $X$ are proximal.

Proposition 8.2. Let $S$ be a semigroup. Then the following are equivalent:

(1) $(M(S), S)$ is rigid, i.e. $|e \cdot M(S)|=1$ for every $e \in \operatorname{Id}(M(S))$;

(2) $(M(S), S)$ is proximal;

(3) every minimal dynamical system for $S$ is proximal;

(4) for any open susbet $U$ of $M(S)$, and any finite collection $\left\{x_{0}, \ldots, x_{n}\right\}$ of points of $M(S)$, there is $s \in S$ so that $s\left(x_{i}\right) \in U$ for all $i \leqslant n$.

Proof. (1) $\rightarrow(2)$ (1) says that $M(S)$ consists of idempotents only. Fix $x, y \in M(S)$. Then $x \cdot x=x \cdot y=x$. Let $\mathcal{C}$ be an open cover of $M(S)$. There are $U \in \mathcal{C}$ and $A \subseteq S$ so that $x \in A^{*} \subseteq U$. Since $x \cdot y=x,\left\{s \in S: s(y) \in A^{*}\right\} \in x$, and since $x \cdot x=x$, $\left\{s \in S: s(x) \in A^{*}\right\} \in x$. It follows that there is $s \in S$ so that $s(x), s(y) \in A^{*} \subseteq U$.

$(2) \rightarrow(1)$ Take an $e \in I d(M(S))$ and $x, y \in e \cdot M(S)$. Let $\mathcal{F}$ be the family of all open covers of $M(S)$. For $P \in \mathcal{F}$ let $X_{P}=\{s \in S:(\exists U \in P)(s(x), s(y) \in U)\}$. If 
$P_{1} \wedge P_{2}$ denotes the common refinement of $P_{1}$ and $P_{2}$, then $X_{P_{1} \wedge P_{2}} \subseteq X_{P_{1}} \cap X_{P_{2}}$, and thus $\left\{X_{P}: P \in \mathcal{F}\right\}$ is centred. Let $p \in \beta S$ be an ultrafilter extending $\left\{X_{P}\right.$ : $P \in \mathcal{F}\}$. Then $p \cdot x=p \cdot y$. Consider the continuous mappings $R_{x}, R_{y}: \beta S \rightarrow \beta S$. $Y=\left\{q \in \beta S: R_{x}(q)=R_{y}(q)\right\}$ is non-empty, as $p \in Y$, and closed in $\beta S$. If $r \in \beta S$ and $q \in Y$, then $R_{x}(r \cdot q)=(r \cdot q) \cdot x=r \cdot(q \cdot x)=r \cdot(q \cdot y)=(r \cdot q) \cdot y=R_{y}(r \cdot q)$, thus, $Y$ is a left ideal of $(\beta S, \cdot)$. Let $M_{1} \subseteq Y$ be a minimal left ideal. Either $M_{1}=M(S)$ and then $R_{x}(e)=R_{y}(e)$, giving $x=y$, or $M_{1} \cap M(S)=\emptyset$ and there is a unique idempotent $e_{1} \in M_{1}$ so that $e \cdot e_{1}=e_{1}$ and $e_{1} \cdot e=e$. Then $R_{x}\left(e_{1}\right)=R_{y}\left(e_{1}\right)$, hence $e_{1} \cdot x=e_{1} \cdot y$. Since $x=e \cdot x$ and $y=e \cdot y$ as both are from $e \cdot M(S)$, it follows that $e_{1} \cdot e \cdot x=e_{1} \cdot e \cdot y$, giving $e \cdot x=e \cdot y$, and so $x=y$.

$(2) \leftrightarrow(3)$ Follows from the fact that a factor of a proximal dynamical system is again proximal.

$(1) \rightarrow(4)$ Let $V$ be an open subset of $M(S)$. Let $\left\{x_{0}, \ldots, x_{n}\right\}$ be an arbitrary finite collection of points of $M(S)$. Pick $y \in V$. Since all elements of $M(S)$ are idempotents, it follows that $y \cdot x_{i}=y$ for any $i \leqslant n$. Then $\left\{s \in S: s\left(x_{i}\right) \in V\right\} \in y$ for all $i \leqslant n$. Thus, there is $s \in S$ so that $s\left(x_{0}\right), \ldots, s\left(x_{n}\right) \in V$.

$(4) \rightarrow(2)$ Take two arbitrary points $x_{1}$ and $x_{2}$ of $M(S)$. Let $\mathcal{C}$ be an open cover of $M(S)$. For some $U \in \mathcal{C}$, there is $s \in S$ so that $s\left(x_{1}\right), s\left(x_{2}\right) \in U$.

Proposition 8.3. Let $S$ be a semigroup so that $M(S)$ has an isolated point. Then the group of automorphisms of $M(S)$ is finite.

Proof. Assume that the group of automorphisms is infinite. Let $p$ be an isolated point of $M(S)$. There is an idempotent $e \in M(S)$, so that $p \in e \cdot M(S)$. Then $e$ is isolated in $M(S)$. It follows that $M(S)=\bigcup_{i \leqslant n} s_{i}^{-1}[\{e\}]$ for some $s_{0}, \ldots, s_{n} \in S$.

Define $P_{i}=e \cdot M(S) \cap s_{i}^{-1}[\{e\}]$, for $i \leqslant n$. By Fact $3.9 e \cdot M(S)$ is isomorphic to the group of automorphisms of $(M(S), S)$, and hence infinite. We can apply Hindman's theorem (see [Hi1]) and so there are $i \leqslant n$ and $a, b \in P_{i}$ so that $a \neq b, a \neq e, b \neq e$, and $a \cdot b \in P_{i}$. Thus $a, b, a \cdot b \in s_{i}^{-1}[\{e\}]$, so $s_{i}(a)=s_{i}(b)=s_{i}(a \cdot b)=e$. It follows that $e=s_{i}(a \cdot b)=s_{i} \cdot(a \cdot b)=\left(s_{i} \cdot a\right) \cdot b=e \cdot b=b$, a contradiction.

\section{REFERENCES}

[A] J. Auslander, Minimal Flows and their Extensions, North-Holland, 1988. MR 89m:54050

[BB] B. Balcar and A. Blaszczyk, On minimal dynamical systems on Boolean algebras, Comment. Math. Univ. Carolinae 31 (1) (1990), 7-11. MR 91h:54059

[BD] B. Balcar and A. Dow, Dynamical systems on compact extremally disconnected spaces, Topology and its Application 41 (1991), 41-56. MR 92k:54048

[BF] B. Balcar and F. Franek, Independent Families in Complete Boolean Algebras, Trans. Amer. Math. Soc. 274 (2) (1982), 607-617. MR 83m:06020

[BJZ] B. Balcar, T. Jech, and J. Zapletal, Semi-Cohen Boolean algebras, Ann. Pure Appl. Logic - submitted.

[BS] B. Balcar and P. Simon, Appendix on General Topology, Handbook of Boolean algebras (J.D. Monk and R. Bonnet, eds.), North-Holland, 1989. MR 90k:06004

[CN] W. W. Comfort and S. Negrepontis, The theory of ultrafilters, Springer-Verlag, Berlin, Heidelberg, 1974. MR 53:135

[CvM] W.W. Comfort and J. van Mill, Some topological groups with, and some without, proper dense subgroups, Topology and its Application 41 (1991), 3-15. MR 92h:54050

[CP] A.H. Clifford and G.B. Preston, The Algebraic Theory of Semigroups, vol. 1, American Mathematical Society, 1961. MR 24:A2627

[vD] E. K. van Douwen, The maximal totally bounded group topology on $G$ and the biggest minimal G-space, for Abelian groups $G$, Topology and its Application 34 (1990), 69-91. MR 91d:54044 
[E] R. Ellis, Lectures on Topological Dynamics, W.A. Benjamin, Inc., 1969. MR 42:2463

[En] R. Engelking, General Topology, PWN, Warsaw, 1977. MR 58:18316b

[F] H. Furstenberg, Recurrence in Ergodic Theory and Combinatorial Number Theory, Princeton University Press, Princeton, New Jersey, 1981. MR 82j:28010

[Fu] S. Fuchino, $\mathcal{L}_{\infty \kappa}$-Cohen algebras, preprint (1992).

[G] S. Glasner, Proximal Flows, Springer-Verlag (Lecture Notes in Mathematics No. 517), 1976. MR 57:13890

[Ga] J. Gait, Transformation groups with no equicontinuous minimal set, Compositio Mathematica 25 (1972), 87-92. MR 47:4233

[H] Handbook of Boolean Algebras (J.D. Monk and R. Bonnet, eds.), North-Holland, 1989. MR 90k:06004

[Hi] N. Hindman, Ultrafilters and Ramsey theory - an update, Set Theory and its Applications Proceedings, Ontario 1987 (J. Steprans and S. Watson, eds.), Springer-Verlag (Lecture Notes in Mathematics No. 1401), 1989. MR 91b:54003

[Hi1] N. Hindman, The existence of certain ultrafilters on $N$ and a conjecture of Graham and Rothschild, Proc. Amer. Math. Soc. 36 (1972), 341-346. MR 46:7041

[HiS] N. Hindman and D. Strauss, Algebraic and Topological Equivalences in the Stone-Čech compactification of a Discrete Senigroup - preprint.

[HS] H. Herrlich and G.E. Strecker, Category Theory, Allyn and Bacon, Inc., Boston, 1973. MR 50:2284

[Ko] S. Koppelberg, A lattice structure on the isomorphism types of complete Boolean algebras, Set Theory and Model Theory, Springer-Verlag (Lecture Notes in Mathematics No. 872), 1981. MR 83b:03005

[Ko1] S. Koppelberg, Characterizations of Cohen algebras, to appear in the proceedings volume of the conference in honour of M. E. Rudin s retirement (Topology conference 1991). MR 95e:06035

[KS] S. Koppelberg and S. Shelah, Subalgebras of Cohen algebras need not be Cohen, preprint.

[L] S. Lang, Algebra, Addison-Wesley, 1984. MR 86j:00003

[P] A.T. Paterson, Amenability, Mathematical Surveys and Monographs (29), American Mathematical Society, 1988. MR 90e:43001

[T] S. Turek, A note on universal minimal dynamical systems, Comment. Math. Univ. Carolinae 32 (4) (1991), 781-783. MR 93f:54053

[deV] J. de Vries, Elements of Topological Dynamics, Kluwer Academic Publishers, 1993. MR 94m:54098

[W] S. Wagon, The Banach-Tarski Paradox, Cambridge University Press, 1985. MR 87e:04007

Mathematical Institute of AV ČR, Žitná 25, 11567 Praha 1, Czech Republic, and Center for Theoretical Study, Jilská 1, 11000 Praha 1, Czech Republic

E-mail address: balcar@beba.cesnet.cz

Department of Computer Science and Systems, McMaster University, Hamilton, OnTARIO, CANAda L8S $4 \mathrm{~K} 1$

E-mail address: franek@mcmail.cis.mcmaster.ca 